\title{
Hydromechanical-Stochastic Modeling of Fluid-Induced Seismicity in Fractured Poroelastic Media ${ }^{1}$
}

leijin@alumni.stanford.edu

\section{Key Points}

- Inter-seismic fracture-poro-mechanics combined with stochastic co-seismic stress drop for modeling fluid-induced seismicity in fractured poroelastic media

- Mechanics-based analysis on the spatial-temporal evolution of seismicity and the associated source parameters

- Seismicity clusters near favorably-oriented large-scale fractures and substantially inhibited by poroelastic coupling in the near field

\section{Abstract}

We present a new method for modeling fluid-perturbation induced seismicity in a fluid-saturated poroelastic medium embedded with a dual network of fractures. The inter-seismic triggering is deterministically modeled using a quasi-static, nonlinear and fluid-solid fully coupled fractureporo-mechanical approach that resolves only the large-scale fractures. The co-seismic dynamic rupture is not explicitly modeled. Instead, the seismicity-induced shear stress drop is approximated as a static quantity and stochastically modeled on a range computed from the evolving poroelastic stress in conjunction with the initial stress and the static and dynamic frictional strengths. These two steps are sequentially connected and then iterated via a predictioncorrection type of fracture stress updating scheme, naturally producing repeating seismic events on certain fractures. As an example, we perform three progressive numerical experiments. By comparing the corresponding synthetic event catalogs, we investigate the effects of fractures and poroelastic coupling on the evolution and source characteristics of the seismicity. Main findings include (1) the seismicity clusters near large-scale fractures favorably oriented and subjected to sufficient perturbations, (2) poroelastic coupling enhances the clustering and substantially inhibits the seismicity in the nearfield and (3) source characteristics and the $b$-value seem not affected by fractures or poroelastic coupling. Our method can serve as a general physics-based tool for more realistically predicting induced seismicity in complex geological media.

\footnotetext{
1 This article is a non-peer reviewed preprint published at EarthArXiv.
} 
31 Keywords: numerical modeling, induced seismicity, discrete fracture network, poroelastic coupling, stress drop, $b$-value

\section{Introduction}

Fluid injection into the subsurface perturbs the pore pressure and alters the effective stress quasistatically, inducing seismicity on fractures of certain orientations (we hereinafter do not distinguish between a fracture and a fault in this study). This process is traditionally considered as a decoupled hydroshear process: the effective normal stress on a fracture decreases by the amount of fluid overpressure according to the simple effective stress law (Terzaghi, 1936), whereas the shear stress remains unchanged (e.g., Byerlee, 1978; Scuderi \& Collettini, 2016; Mukuhira et al, 2017), resulting in a direct increase in the Coulomb stress, which, when driven from negative to zero, signifies the occurrence of seismicity. Such a decoupled mechanism remains as the basis of some prevalent statistical models of induced seismicity in a permeable porous medium (e.g., Shapiro et al., 2005; Rothert \& Shapiro, 2007). In this class of models, a statistically random critical pore pressure is used as a proxy of the frictional strength of a preexisting fracture and the pore pressure evolution is governed by simple linear fluid diffusion; the modeled spatial-temporal distribution of seismicity, however, is often inconsistent with observations. As a remedy, some nonlinear diffusion models have been developed by adding a pressure-dependent diffusivity (Hummel \& Shapiro, 2012; Johann et al., 2016; Carcione et al., 2018). The diffusion-based seismicity models can be further extended by incorporating, e.g., random stress heterogeneity (Goertz-Allmann \& Wiemer, 2012), fractures following distributions derived from field observations (Verdon et al., 2015), and even empirical seismic emission criteria for generating synthetic seismograms (Carcione et al., 2015). This decoupled mechanism also underlies some studies that invert for distributions of permeability (Tarrahi \& Jafarpour, 2012) and pore pressure (Terakawa et al., 2012; Terakawa, 2014) from induced seismicity data.

However, the decoupled mechanism inherently cannot explain the remoting triggering of seismicity in areas not subjected to pressure perturbation (Stark \& Davis, 1996; Megies \& Wassermann, 2014; Yeck et al., 2016); it also directly contradicts the commonly observed depletion-induced seismicity (Zoback \& Zinke, 2002). Motivated by such field evidences, a large body of analytical solutions (Segall, 1985; Segall et al., 1994; Segall \& Fitzgerald, 1998; Altmann et al., 2014; Segall \& Lu, 2015; Jin \& Zoback, 2015a) and numerical solutions (Murphy et al., 2013; Chang \& Segall, 2016a; Chang \& Segall, 2016b; Chang \& Segall, 2017; Fan et al., 2016; Deng et al., 2016; Zbinden et al., 2017) have been proposed, providing poroelastic models of induced seismicity. At a smaller scale, numerical simulations of fluid-induced microseismicity, typically motivated by applications to the stimulation of hydrocarbon and geothermal reservoirs, have also been reported (e.g., Maillot et al., 1999; Baisch et al., 2010; Wassing et al., 2014; Zhao \& Young, 
scale of interest, these studies substantiate that poroelastic coupling may play an important role in inducing seismicity.

Despite these evidences, some debates persist, mainly from those who advocate the simple diffusion-only models (Johann et al., 2016). They claim that their diffusion models approximate poroelastic models if the Biot-Willis coefficient $a$ is small; they also argue that when $a$ is less than 0.3 , it is the pore pressure rather than the poroelastic stress that dominates the hydroshear on fractures; they further question the Segall $(2015,2016 a)$ poroelastic models in which $a$ is greater than 0.3, and hypothesize that for nearly impermeable rocks, a should also be negligible. However, one must realize that $a$ is a measurement of the rock solid's susceptibility to the influence of the fluid and vice versa; it is not a property directly related to the permeability of the rock itself. As a matter of fact, laboratory experiments show that $a$ of unconventional reservoir rocks is indeed primarily between 0.3 and 0.9 (e.g., Ma \& Zoback, 2017).

Some other studies seek middle ground by considering the co-existence of the pore pressure effect and the poroelatic effect such that induced seismicity is a result of both (e.g., Barbour et al., 2017; Keranen \& Weingarten, 2018). This is perhaps a misconception. Jin \& Zoback (2017) demonstrated the fundamental difference between the two, which lies in how the fluid overpressure modifies the effective stress tensor that will be used for calculating stress on a fracture. Using the Biot effective stress law (Biot, 1941) as an example, the pore pressure effect is stated as:

whereas the poroelastic effect, more precisely, the fluid-to-solid coupling effect, arise from solving the following conservation law:

$$
\boldsymbol{\sigma}_{p}{ }^{\prime}+\alpha p \mathbf{1}=\mathbf{0}
$$

$$
\nabla \cdot\left(\boldsymbol{\sigma}_{p}{ }^{\prime}+\alpha p \mathbf{1}\right)=0
$$

Here in equations (1) and (2), $p$ is the fluid overpressure, $\boldsymbol{\sigma}_{p}{ }^{\prime}$ is the associated change in the effective stress tensor (both are quasi-static perturbations to their respective initial reference state) and $\mathbf{1}$ is the Kronecker delta. $\boldsymbol{\sigma}_{p}{ }^{\prime}$ is the fundamental reason driving changes in the stress on a fracture and inducing seismicity. Since the linear momentum should be always conserved between the perturbations, one must solve for $\boldsymbol{\sigma}_{p}{ }^{\prime}$ from equation (2) instead of simply assuming $\boldsymbol{\sigma}_{p}{ }^{\prime}=-a p \mathbf{1}$ as is stated by equation (1). As a matter of fact, $\mathbf{\sigma}_{p}{ }^{\prime} \neq-a p \mathbf{1}$ as long as $p$ is not spatially uniform (i.e., a pressure gradient is present, $\nabla p \neq 0$ ). For any fluid saturated media, the poroelastic coupling effect is the true and only effect; the pore pressure effect is the 'reduced' poroelastic effect when the pressure gradient vanishes and the two should not be considered as co-existing effects.

Poroelastic coupling is undoubtedly the mechanism behind induced seismicity. However, the exact role is plays and its influence on the source characteristics remain somewhat unclear. 
101 Furthermore, the aforementioned poroelastic models only include fractures very limited in 102 distribution, therefore, the role of fractures cannot be sufficiently explored, neither. The fractures 103 are also explicitly represented as entities following the same fluid and solid rheologies as the 104 hosting rock, therefore, the medium is effectively 'porous' only. Such simplifications may come with certain consequences. Some studies suggest that accounting for both poroelastic coupling and an arbitrary discrete fracture network (DFN) permitted to have different constitutive behaviors can lead to radically different modeling outcomes (Jin \& Zoback, 2016a; Jin \& Zoback, 2016b). Pertaining to this issue, some studies resolve very regularly distributed fractures (e.g., Safari \& Ghassemi, 2016); others attempt to include an arbitrary DFN, among which, e.g., some focus on the fluid pressure and solid deformation only within fractures but not the hosting rock (Farmahini-Farahani \& Ghassemi, 2016), some consider coupling only upon the occurrence of seismicity (Bruel, 2007). None of these models produces repeating events frequently detected in catalogs of induced seismicity (e.g., Baisch \& Harjes, 2003; Moriya et al., 2003; Deichmann et al., 2014; Duverger et al, 2015).

To date, a general method for modeling fluid-induced seismicity accounting for arbitrary fractures and poroelastic coupling is lacking. We are therefore motivated to develop the following new method aimed for a fractured poroelastic medium. It combines the deterministic modeling of inter-seismic, quasi-static and hydromechanically coupled triggering and the stochastic modeling of co-seismic shear stress drop, both repeated over multiple seismic cycles. It is capable of not only realistically predicting the spatial-temporal evolution of seismicity but also generating a synthetic event catalog that allows for the exploration of the role of model physics as well as their connections to observations. Details are described below.

\section{Methodology}

\subsection{Sources of Fracture Stress}

125 Given a location $\underline{x}$ and a time $t$ over $\Omega \times[0, \mathrm{~T}]$ where $\Omega$ is the domain of interest and [0,T] is the 126 time interval, the effective stress tensor $\boldsymbol{\sigma}^{\prime}(\underline{x}, t)$ in a fluid-saturated poroelastic medium undergoing seismicity can be decomposed as the following:

$$
\boldsymbol{\sigma}^{\prime}(\underline{x}, t)=\boldsymbol{\sigma}_{0}{ }^{\prime}(\underline{x})+\boldsymbol{\sigma}_{p}{ }^{\prime}(\underline{x}, t)+\sum_{j} \boldsymbol{\sigma}_{s}{ }^{\prime}\left(\underline{x}, t_{j}^{*}+\delta t_{j}\right)
$$

where $\boldsymbol{\sigma}_{0}{ }^{\prime}(\underline{x})$ is the initial in-situ effective stress tensor, $\boldsymbol{\sigma}_{p}^{\prime}(\underline{x}, t)$ is the fluid perturbation-induced effective stress tensor relative to $\boldsymbol{\sigma}_{0}{ }^{\prime}(\underline{x})$ (same as in equation (1) and (2)) and $\boldsymbol{\sigma}_{s}{ }^{\prime}\left(\underline{x}, t_{j}{ }^{*}+\delta t_{j}\right)$ is the slipinduced change in the effective stress tensor over the $j^{\text {th }}$ co-seismic interval where $t_{j}{ }^{*}$ and $\delta t_{j}$ are

132 the associated beginning time and duration. $\boldsymbol{\sigma}_{0}{ }^{\prime}(\underline{x})$ is time-independent and in principle permits heterogeneity; $\operatorname{tr}\left(\boldsymbol{\sigma}_{p}{ }^{\prime}(\underline{x}, t)\right)$ (the diagonal sum) is fully coupled with the negative gradient of the associated fluid pressure $p(\underline{x}, t)$ and the two must be solved for simultaneously; in $\boldsymbol{\sigma}_{s}{ }^{\prime}\left(\underline{x}, t_{j}^{*}+\delta t_{j}\right)$, 
$\delta t_{j} \ll t$ such that relative to the time scale relevant to a complete seismic cycle, $\delta t_{j} \approx 0$ and $\boldsymbol{\sigma}_{s}{ }^{\prime}\left(\underline{x}, t_{j}{ }^{*}+\delta t_{j}\right)$ can be approximated as a static quantity:

$$
\boldsymbol{\sigma}_{s}{ }^{\prime}\left(\underline{x}, t_{j}^{*}+\delta t_{j}\right) \approx \boldsymbol{\sigma}_{s j}{ }^{\prime}(\underline{x})
$$

The stress on a fracture intersecting $\underline{x}$ and at $t$ is given by:

$$
\sigma_{n}^{\prime}(\underline{x}, t)=\boldsymbol{\sigma}^{\prime}(\underline{x}, t): \underline{n} \otimes \underline{n}
$$

$$
\tau(\underline{x}, t)=\left[\left\|\boldsymbol{\sigma}^{\prime}(\underline{x}, t) \cdot \underline{n}\right\|^{2}-\left(\boldsymbol{\sigma}^{\prime}(\underline{x}, t): \underline{n} \otimes \underline{n}\right)^{2}\right]^{\frac{1}{2}}
$$

$$
C F F(\underline{x}, t)=\tau(\underline{x}, t)-\mu_{s} \sigma_{n}^{\prime}(\underline{x}, t)
$$

In equations (5) - (7), $\sigma_{n}{ }^{\prime}(\underline{x}, t), \tau(\underline{x}, t)$ and $C F F(\underline{x}, t)$ are the effective normal stress, the shear stress and the Coulomb Failure Function (i.e., the Coulomb stress, $\leq 0$ ) on the fracture of interest, and $\underline{n}$ and $\mu_{s}$ are the unit normal vector and the static frictional coefficient of the fracture.

Equations (3) - (7) show that $\boldsymbol{\sigma}_{p}{ }^{\prime}(\underline{x}, t)$ and $\boldsymbol{\sigma}_{s j}{ }^{\prime}(\underline{x})$ are the two primary sources driving changes in the stress on a fracture. In general, for $\sigma_{n}{ }^{\prime}(\underline{x}, t), \mathbf{\sigma}_{p}{ }^{\prime}(\underline{x}, t)$ can either increase or decrease it whereas $\boldsymbol{\sigma}_{s j}{ }^{\prime}(\underline{x})$ causes minor variations to it except near fracture tips; for $\tau(\underline{x}, t), \boldsymbol{\sigma}_{p}{ }^{\prime}(\underline{x}, t)$ compensates, albeit possibly negatively depending on the configuration, the fracture for the shear stress loss resulting from $\boldsymbol{\sigma}_{s j}{ }^{\prime}(\underline{x})$.

To model induced seismicity in a fractured poroelastic medium, one must go through equations (3)-(7) and check $C F F(\underline{x}, t)$ against 0 to determine if seismicity occurs; if yes, the effective stress tensor needs be updated $(j=j+1)$ for the next seismic cycle. This process needs to be repeated iteratively for all fractures at all time steps. For a given fracture that has undergone at least one seismic cycle, equations (3)-(6) yield a complete stress path associated with this cycle in the fracture effective normal stress-shear stress space. CFF remains constrained below 0 throughout the process.

The major computational cost then arises from the calculation of $\boldsymbol{\sigma}_{p}{ }^{\prime}(\underline{x}, t)$ over the quasi-static inter-seismic (i.e., pre-seismic and post-seismic) phase and $\boldsymbol{\sigma}_{s}{ }^{\prime}\left(\underline{x}, t_{j}{ }^{*}+\delta t_{j}\right)\left(\right.$ or $\left.\boldsymbol{\sigma}_{s j}{ }^{\prime}(\underline{x})\right)$ over the coseismic phase. Notice these two quantities can be solved for separately if assuming a linearly elastic solid irrespective of the fluid which can behave either linearly or nonlinearly. The former can be sufficiently addressed using our Jin \& Zoback (2017) computational model; for a detailed description on the latter process resulting from a fully dynamic and spontaneously rupturing seismic event while considering the effect of $\boldsymbol{\sigma}_{p}{ }^{\prime}(\underline{x}, t)$, we refer the reader to Jin \& Zoback (2018a, 2018b). In this study, we are concerned only with the inter-seismic evolution of induced seismicity but not the co-seismic dynamic changes (i.e., wave propagation), therefore, instead of solving for both $\boldsymbol{\sigma}_{p}{ }^{\prime}(\underline{x}, t)$ and $\boldsymbol{\sigma}_{s}{ }^{\prime}\left(\underline{x}, t_{j}{ }^{*}+\delta t_{j}\right)$ for updating the fracture stress, we will instead solve only for $\boldsymbol{\sigma}_{p}{ }^{\prime}(\underline{x}$, 
167 t) and then insert it into a stress updating algorithm to indirectly account for seismicity-induced stress changes on a fracture. The details of these two steps are given in the following two sections.

Table 1. Three progressive cases

\begin{tabular}{ll}
\hline Case & Governing equations \\
1 & equations (A7), (A3) \\
2 & equations (A1), (A3), (A4) \\
3 & equations (A1)-(A5)
\end{tabular}
expressed as:

\subsection{Fracture-Poro-Mechanical Modeling}

The objective here is to calculate $\boldsymbol{\sigma}_{p}^{\prime}(\underline{x}, t)$ as an input for updating the fracture stress. As mentioned above, $\mathbf{\sigma}_{p}^{\prime}(\underline{x}, t)$ must be solved for together with the associated fluid pressure $p(\underline{x}, t)$ in a fully coupled manner. Aside from the full coupling itself, another major challenge lies in that both are a function of the arbitrary network of pre-existing fractures spanning over a wide range of scales. While accounting for all fractures is probably computationally intractable, the subset of fractures at a scale comparable to the size of the model domain of interest must be deterministically resolved, as they have amply been demonstrated to have a first-order control of the modeling outcome (Berkowitz, 2002; Vujevic' et al., 2014; Hirthe \& Graf, 2015; Hardebol et al., 2015). We hereinafter refer to these fractures as the large-scale deterministic fractures (LSDF), which can be

where $F_{I}$ is the $I^{\text {th }}$ large-scale fracture and $N$ is the total number of large-scale fractures.

We will also refer to the step solving for the fully coupled $\boldsymbol{\sigma}_{p}^{\prime}(\underline{x}, t)$ and $p(\underline{x}, t)$ by considering the LSDF as the fracture-poro-mechanical modeling. Within the framework of Biot's theory of poroelasticity, Jin \& Zoback (2017) formulated the problem of fluid-solid fully coupled quasistatic poromechancis of an arbitrarily fractured and deformable porous solid saturated with a single-phase compressible fluid. Several key governing equations together with a brief description can be found in appendix A.1. This model is adopted here. To investigate the effect of the LSDF and the effect of poroelastic coupling on seismicity, we construct the following three progressive cases, each physically more representative than the previous, see Table 1.

(adding the effect of poroelastic coupling to case 2)

Case 1 states a standard fluid-diffusion problem in a fluid-saturated porous medium; case 2 is similar to case 1 except for the addition of the LSDF contributing to the fluid-diffusion; case 3 describes an otherwise complete poroelastic problem in a fractured medium except for the exclusion of equation (A6), which can render the modeled stress highly heterogeneous characterized by concentration, compartmentalization and apparent discontinuities (Jin \& 
Zoback, 2017). To single out the effect of poroelastic coupling, equation (A6) is not considered in this study such that meaningful comparisons can be made between cases 2 and 3 .

In seeking for a numerical solution, Jin \& Zoback (2017) developed a hybrid-dimensional twofield mixed finite element method for efficient space discretization while preserving the distribution of a given set of deterministic fractures; the solution of the fully coupled semidiscrete system is advanced in time in a fully coupled manner (as opposed to a sequentially coupled manner) following a fully implicit (backward Euler) finite difference scheme; within each time step, the resulting nonlinear and fully discrete equation is solved using a Newton-Raphson solver. This technique is adopted for case 3. For case 1 , the discretization is done in space using a standard Galekin finite element method and in time using a backward Euler scheme; no linearization is needed. For case 2, the discretization and linearization procedures resemble those in case 3 except for the use of a single-field interpolation scheme. To illustrate the differences, for cases 1-3, we give their respective semi-discrete form of the governing laws shown in Table 1 after space discretization. They read:

$$
\begin{gathered}
\tilde{\mathbf{M}}_{m} \dot{\hat{\zeta}}_{m}+\mathbf{K}_{m} \hat{\zeta}_{m}=\underline{F}_{1} \\
\left(\mathbf{M}_{m}+\sum_{I}^{N} \mathbf{M}_{F_{I}}\left(\hat{\zeta}_{F_{I}}\right)\right) \dot{\hat{\zeta}}_{m}+\left(\mathbf{K}_{m}+\sum_{I}^{N} \mathbf{K}_{F_{I}}\left(\hat{\zeta}_{F_{I}}\right)-\sum_{J}^{N_{I}} \mathbf{K}_{m F_{J}}\left(\hat{\zeta}_{F_{J}}\right)+\sum_{K}^{N_{I}} \mathbf{K}_{F_{K} m}\right) \hat{\zeta}_{m}=\underline{F}_{2} \\
{\left[\begin{array}{cc}
\mathbf{M}_{m}+\sum_{I}^{N} \mathbf{M}_{F_{I}}\left(\hat{\zeta}_{f_{I}}\right) & -\mathbf{C}^{T} \\
\mathbf{0} & \mathbf{0}
\end{array}\right]\left\{\begin{array}{l}
\dot{\hat{\zeta}}_{m} \\
\underline{\dot{d}}_{m}
\end{array}\right\}+\left[\begin{array}{rr}
\mathbf{K}_{m}+\sum_{I}^{N} \mathbf{K}_{F_{I}}\left(\hat{\zeta}_{F_{I}}\right)-\sum_{J}^{N_{I}} \mathbf{K}_{m F_{J}}\left(\hat{\zeta}_{F_{J}}\right)+\sum_{K}^{N_{H}} \mathbf{K}_{F_{K} m} & \mathbf{0} \\
\mathbf{C} & \mathbf{G}_{m}
\end{array}\right]\left\{\begin{array}{l}
\hat{\zeta}_{m} \\
\underline{d}_{m}
\end{array}\right\}=\left\{\begin{array}{l}
\underline{F}_{3} \\
\underline{Y}
\end{array}\right\}}
\end{gathered}
$$

where $\widetilde{\mathbf{M}}$ and $\mathbf{M}$ are the fluid storage capacity matrix without and with the presence of fractures, $\mathbf{K}$ is the hydraulic conductivity/transferability matrix, $\mathbf{G}$ is the stiffness matrix, $\mathbf{C}$ is the coupling matrix, $\underline{F}_{1}, \underline{F}_{2}$ and $\underline{F}_{3}$, which take different forms, are the external nodal mass for cases $1-3, \underline{Y}$ is the external nodal force, $\hat{\zeta}$ and $\underline{d}$ are the nodal fluid pressure and solid displacement vectors, subscripts ' $m$ ' and ' $F$ ' indicate quantities associated the porous matrix and the LSDF, subscripts ' $m F^{\prime}$ and ' $F m^{\prime}$ indicate matrix-to-fracture and fracture-to-matrix interactions, $I$ and $N$ are the same as in equation (8), and $J$ and $K$ are the index of a fracture within the so-called type- $I$ and type-II subsets and $N_{I}, N_{I I}$ are the respective number of fractures, $N_{I}+N_{I I}=N$. The detailed expressions of the above matrices and vectors can be found in Jin \& Zoback (2017). $\widetilde{\mathbf{M}}, \underline{F}_{1}, \underline{F}_{2}$ can be obtained by removing the fracture effect and/or the coupling effect from their respective counterparts.

Solving the respective fully discrete form of equations (9)-(11) allows us to calculate $\boldsymbol{\sigma}_{p}{ }^{\prime}(\underline{x}, t)$ as an input for the subsequent seismicity modelling. For cases 1 and $2, \mathbf{\sigma}_{p}{ }^{\prime}(\underline{x}, t)$ is in a standard tensor notation and it reads, following a compressive stress/ pressure positive notation as is used in this study, the following: 


$$
\boldsymbol{\sigma}_{p}{ }^{\prime}(\underline{x}, t)=-\alpha \hat{\zeta}(\underline{x}, t) \mathbf{1}
$$

228

where $\alpha$ is the Biot-Willis coefficient, and $\mathbf{1}$ is the Kronecker delta (see also appendix A.1). and for case $3, \boldsymbol{\sigma}_{p}{ }^{\prime}(\underline{x}, t)$ is in the so-called Voigt notation and it is calculated from $\underline{d}(\underline{x}, t)$ as:

$$
\boldsymbol{\sigma}_{p}{ }^{\prime}(\underline{x}, t)=\mathbf{D B} \underline{d} \underline{x}(\underline{x}, t)
$$

where $\mathbf{B}$ is standard displacement-strain transformation matrix and $\mathbf{D}$ is the elastic stiffness matrix.

\subsection{Seismicity Modeling}

The main task here is to update the stress on fractures resulting from $\boldsymbol{\sigma}_{p}{ }^{\prime}(\underline{x}, t)$ and, if seismicity occurs, from $\boldsymbol{\sigma}_{s j}{ }^{\prime}(\underline{x})$. To do so, we consider a dual network of fractures, hereinafter referred to as the $D F$. It consists of two complementary subsets $\mathrm{A}$ and $\mathrm{B}$, where the subset $\mathrm{A}$, denoted as $\overline{L S D F}$, is an approximation to the $L S D F$ using a series of discrete fractures and the subset $\mathrm{B}$ is a stochastic representation of small-scale fractures typically found in the surrounding hosting rock and is hereinafter referred to as the SSSF. The above description can be summarized as:

$$
D F=\widetilde{\operatorname{LSDF}} \bigcup \operatorname{SSSF}=\left(\bigcup_{a}^{n_{A}} f_{a}\right) \cup\left(\bigcup_{b}^{n_{B}} f_{b}\right)
$$

where $f_{a}$ is the $a^{\text {th }}$ fracture in the subset $\mathrm{A}, f_{b}$ is the $b^{\text {th }}$ fracture in the subset $\mathrm{B}$, and $n_{a}$ and $n_{b}$ are the respective total number of fractures.

The DF given by equation (14) is used for the seismicity modeling. For the reasons explained in section 2.1, we will update the fracture stress first using only $\boldsymbol{\sigma}_{p}{ }^{\prime}(\underline{x}, t)$ and then correct for changes due to $\boldsymbol{\sigma}_{s j}{ }^{\prime}(\underline{x})$. To do so, we make three assumptions. First, fracture slip causes negligible changes in the effective normal stress on the fracture. This is an acceptable assumption for the area on the fracture not immediately near its tips. From equation (5), this reads:

$$
\boldsymbol{\sigma}_{s j}^{\prime}(\underline{x}): \underline{n} \otimes \underline{n} \approx 0
$$

Equation (15) implies that,

$$
\boldsymbol{\sigma}^{\prime}(\underline{x}, t): \underline{n} \otimes \underline{n} \approx\left(\boldsymbol{\sigma}_{0}^{\prime}(\underline{x})+\boldsymbol{\sigma}_{p}^{\prime}(\underline{x}, t)\right): \underline{n} \otimes \underline{n}
$$

On the other hand, the shear stress on the fracture stated by equation (6), when accounting for the effect of $\boldsymbol{\sigma}_{s j}{ }^{\prime}(\underline{x})$, can be re-written in the following form:

$$
\sqrt{\left\|\boldsymbol{\sigma}^{\prime}(\underline{x}, t) \cdot \underline{n}\right\|^{2}-\left(\boldsymbol{\sigma}^{\prime}(\underline{x}, t): \underline{n} \otimes \underline{n}\right)^{2}}=\sqrt{\left\|\left(\boldsymbol{\sigma}_{0}{ }^{\prime}(\underline{x})+\boldsymbol{\sigma}_{p}{ }^{\prime}(\underline{x}, t)\right) \cdot \underline{n}\right\|^{2}-\left(\left(\boldsymbol{\sigma}_{0}{ }^{\prime}(\underline{x})+\boldsymbol{\sigma}_{p}{ }^{\prime}(\underline{x}, t)\right): \underline{n} \otimes \underline{n}\right)^{2}}-\sum_{j} \Delta \tau_{j}
$$


Here, $\Delta \tau_{j}$ is the shear stress drop on the fracture due to the $j^{\text {th }}$ co-seismic interval. Our second assumption reads:

$$
\Delta \tau_{j}=r \Delta \tau_{j \max }
$$

Here,

$$
\Delta \tau_{j \max }=\left(\mu_{s}-\mu_{d}\right)\left(\boldsymbol{\sigma}_{0}^{\prime}(\underline{x})+\boldsymbol{\sigma}_{p}{ }^{\prime}\left(\underline{x}, t_{j}^{*}\right)\right): \underline{n} \otimes \underline{n}
$$

In equations (18) and (19), $\mu_{d}$ is the dynamic frictional coefficient of the fracture as is typically used in a slip-weakening law (Andrews, 1976), $\Delta \tau_{j \max }$ is the maximum likely shear stress drop and $r$ is a stochastic parameter between 0 and 1 to account for the potential non-full degree of shear stress drop (see also Verdon et al., 2015). In this study, we let the probability density function of $r$ follow a uniform distribution. Equations (18) and (19) state that (1) the new shear stress on a fracture due to seismicity is constrained above a lower bound defined by the residual frictional strength of the fracture and (2), more importantly, the maximum likely shear stress drop is dictated by the evolution of the poroelastic stress. This is different from directly prescribing the shear stress drop (e.g., Izadi \& Elsworth, 2014).

Based on the above two assumptions, we propose the following incremental fracture stress updating algorithm, as is shown in List 1.

List 1. Incremental fracture stress updating algorithm for the seismicity modeling

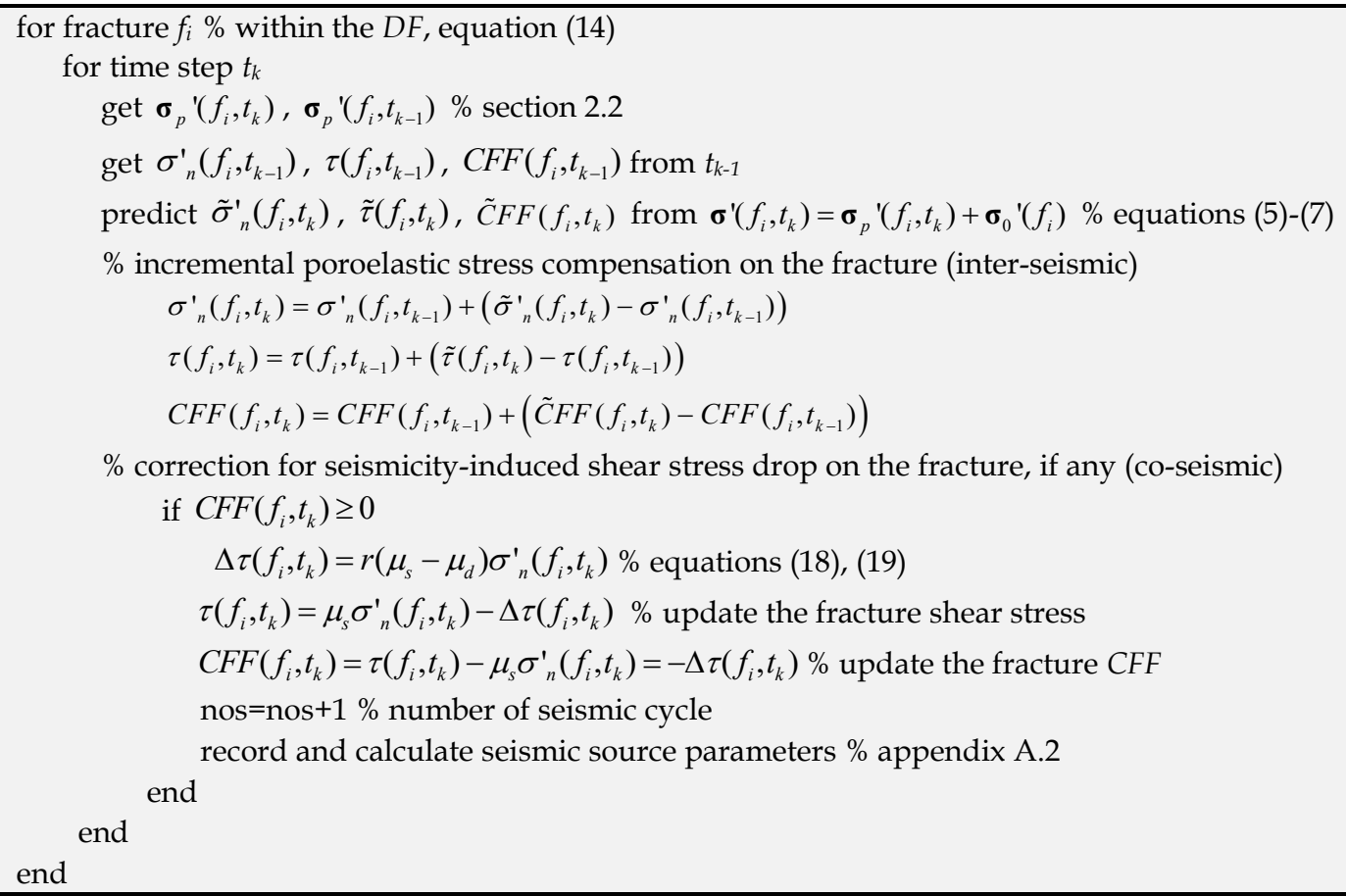


In List 1 , the fracture $f_{i}$ needs to be associated with a stress tensor $\mathbf{\sigma}_{p}{ }^{\prime}\left(f_{i}, t\right)$. Since $f_{i}$ can intersect multiple elements (or Gauss integration points if using high-order finite elements), as the third assumption, we will use only the stress tensor from the element nearest to its center. The above algorithm automatically considers multiple seismic cycles and therefore is naturally capable of modeling repeating seismic events. We are now at a place to model fluid-induced seismicity in a fluid-saturated and fractured poroelastic medium, see Figure 1 for a schematic illustration. A complete seismicity catalog containing information on, e.g., the event origin time $t_{0}$, the location $\underline{x}$, the shear stress drop $\Delta \tau$, the seismic moment $M_{0}$, the moment magnitude $M_{\mathrm{w}}$, the fracture length $L$, the initial Coulomb stress $C F F_{0}$ and the permeability change $k^{*}$, can be assembled. Several key equations for calculating these parameters are outlined in appendix A.2. Notice in equation (A8), a unit length along the third dimension is used. Additionally, the definitions of a triggered event and an induced event are given in appendix A.3 and they will be used later for classifying the modeled events.

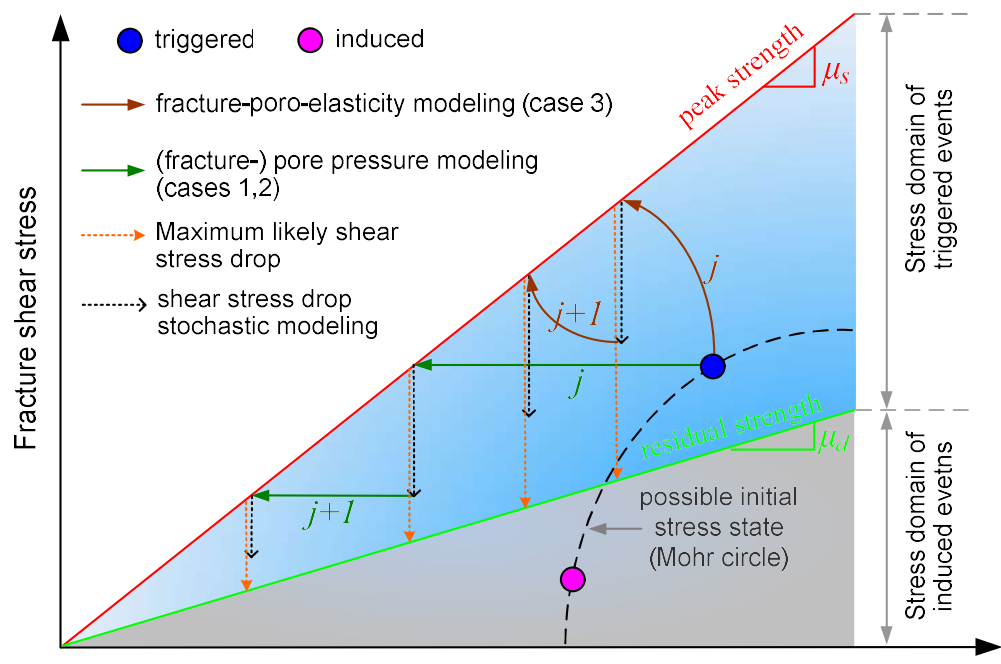

Fracture effective normal stress

Figure 1. Schematic illustration (not to scale) of the hydromechanical-stochastic modeling of fluid-induced seismicity in a fluid-filled and fractured poroelastic medium plotted in the fracture effective normal stress-shear stress space. Based on the peak and residual frictional strengths of a fracture, as are depicted by the red and green lines, the space is divided into two parts defining the initial stress domain for a triggered event and an induced event, respectively. The blue and magenta dots are given as two examples, both located on a Mohr circle defined by $\boldsymbol{\sigma}_{0}{ }^{\prime}(\underline{x})$. For either type of event, the seismicity modeling consists of two steps. The first step is to predict the fracture stress by compensating the fracture with $\boldsymbol{\sigma}_{p}{ }^{\prime}(\underline{x}, t)$, which requires the pore pressure modeling for case 1 , the fracture-pore pressure modeling for case 2 and the fracture-poro-mechanical modeling for case 3, the latter two resolving the LSDF. The outcome of this step is indicated by the green and red arrows. The second step, which does not vary among the three cases, is to stochastically model $\Delta \tau$ on the fracture as indicated by the dashed arrows to approximately account for the effect of $\boldsymbol{\sigma}_{s}{ }^{\prime}(\underline{x})$; meanwhile, $\Delta \tau$ remains constrained on a range $\Delta \tau_{\max }$ as is indicated by the yellow arrows and it is computed from $\boldsymbol{\sigma}_{p}{ }^{\prime}(\underline{x}, t)$ in conjunction with $\boldsymbol{\sigma}_{0}{ }^{\prime}(\underline{x})$. Two consecutive seismic cycles $j$ and $j+1$ are shown, and the complete stress updating scheme is given in List 1. 


\section{Model Set-up}

\subsection{Step 1 for Fracture-Poro-Mechanical Modeling}

As a numerical example, we construct a $200 \mathrm{~m} \times 200 \mathrm{~m}$ 2D domain representing a fracture-hosting porous rock. For cases 2 and 3, we resolve a LSDF with 100 members with their length ranging from $20 \mathrm{~m}$ to $50 \mathrm{~m}$, and orientation, from 0 to $360^{\circ}$, see Figure $2 \mathrm{a}$. The model domain is then discretized in space, see Figure $2 b$, to arrive at the semi-discrete forms given by equations (10) and (11). For case 1, no fracture is present; nevertheless, for meaningful comparisons, the same mesh is used for arriving at equation (9). For cases 2 and 3, the nominal model parameters, including the hydraulic and mechanical properties, the coupling coefficient (i.e., the Biot-Willis coefficient $\alpha$ ), the fluid and solid boundary values and the time-stepping parameter are identical to those in Jin \& Zoback (2017). Of our particular interest is the hydraulic diffusivity of the hosting rock and the $L S D F$ in cases 2 and 3, which are $9.95 \times 10^{-4} \mathrm{~m}^{2} / \mathrm{s}$ and $6.64 \mathrm{~m}^{2} / \mathrm{s}$, respectively. For case $\mathrm{mD}$, leading to a hydraulic diffusivity $D_{h}=0.03 \mathrm{~m}^{2} / \mathrm{s}$. The rationale behind the choice of this number is explained in section 4.3. For all cases, a plane strain assumption is made.
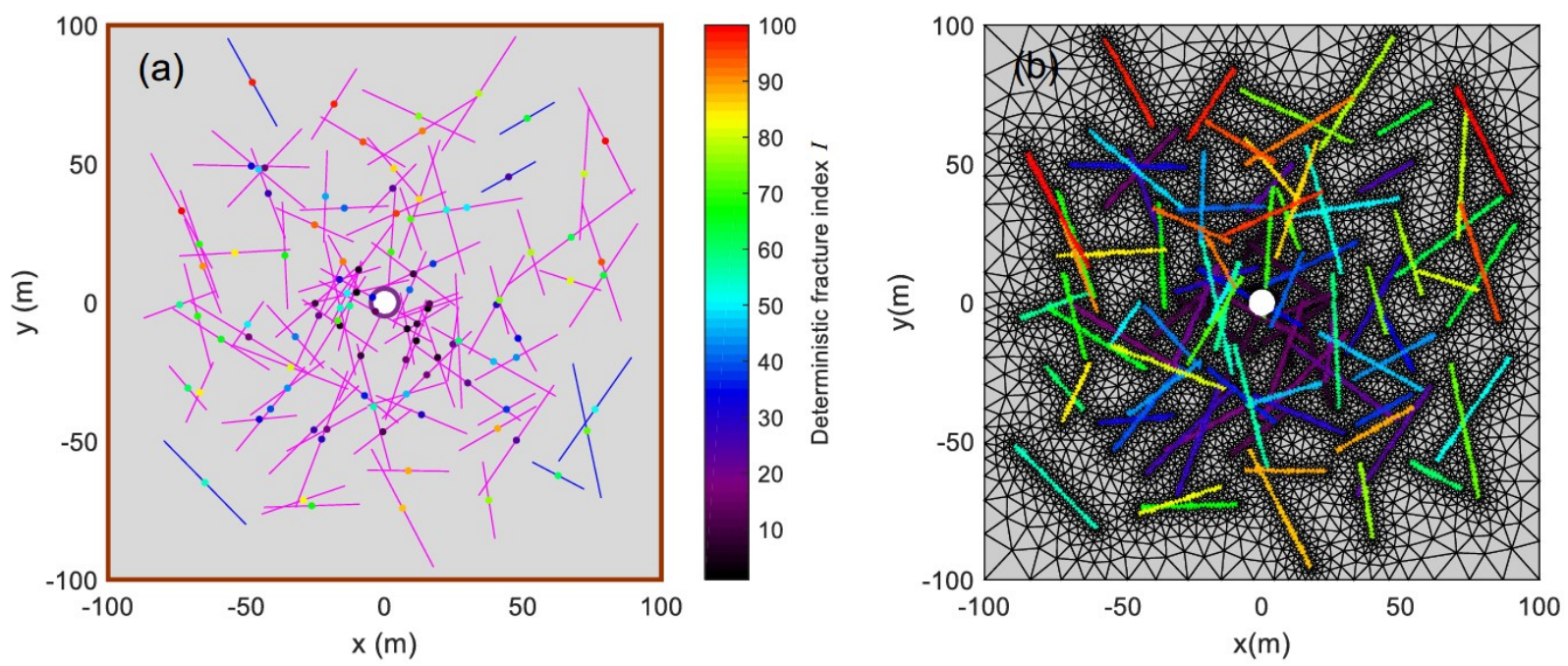

Figure 2. (a) The model domain for cases 2 and 3. It consists of a LSDF embedded within an otherwise porous matrix. Each dot represents the center of the associated fracture, and the color suggests the index I (see equation (8)). Magenta and blue lines represent interconnected and isolated fractures in relation to the fluid boundaries (or the external fluid source) as are depicted by the purple circle and the dark red lines; they require different treatment of the mass exchange with the surrounding matrix. For case 1, the $L S D F$ is removed from the domain. (b) Conforming space discretization of the fractured domain and the resulting unstructured triangular finite elements used in arriving at the semi-discrete forms. For case 3, all elements represent the porous hosting rock; the grey elements are the standard two-field (fluid pressure, solid displacement) mixed FE elements; the colored elements are 'hybrid' mixed elements in which at least one edge is also used as a lower-dimensional element to discretize the fractures; the color of an element indicates the $I^{\text {th }}$ deterministic fracture with which it is associated. If a hybrid element conforms to multiple fractures, only the largest 
only used for interpolating the fluid pressure). For case 1, all elements are the standard single-field elements. Adapted from Jin \& Zoback (2017).

\subsection{Step 2 for Seismicity Modeling}

The next step is to set up the DF for the seismicity modeling, see Figure 3, and this involves two sub-steps, see equation (14). Take cases 2 and 3 for example, the first sub-step is to approximate the $L S D F$ shown in Figure 2a with a $\widetilde{L S D F}$ as the subset A, see Figure 3a, by honoring the original locations and orientations. The second sub-step is to construct a SSSF in the hosting rock as the subset B, see Figure 3b; in principle, this can be derived from a statistical model if data is available. In this example, for simplicity and this does not change the generality of our method, we assign only one fracture to each element center shown in Figure $2 b$ as the modeling of fracture locations; for subset $\mathrm{A}$, the orientations are the same as the associated deterministic fracture; for subset $\mathrm{B}$, the orientations are randomly generated following a uniform distribution on $\left[0,360^{\circ}\right]$. Subsets $A$ and B constitute the complete DF for the seismicity modeling, see Figure 3c. In this process, the fracture length is generated by obeying the following well-established scaling relation, which states that the number of fractures within a natural fracture system scales with the fracture length according to a power law (e.g., Bonnet et al., 2001; Johri \& Zoback, 2014; Jin \& Zoback, 2015b):

$$
N=C L^{-D}
$$

where $N$ is the number of fractures of length $L, C$ is a site-specific constant and $D$ is the so-called fractal dimension and a typical value is between 1 and 2 . In this study, $C=1.6861$ and $D=1.0015$ (further details in section 4.5.2).

The generated $L$ is randomly distributed to all fractures shown in Figure 3c. For case 1, the above two sub-steps are repeated, however, in the first sub-step, the fracture orientations no longer honor the original ones. The resulting two subsets of fractures are shown in Figures $3 \mathrm{~d}$ and $3 \mathrm{e}$ and the complete $D F$ is shown in Figures $3 \mathrm{f}$. 

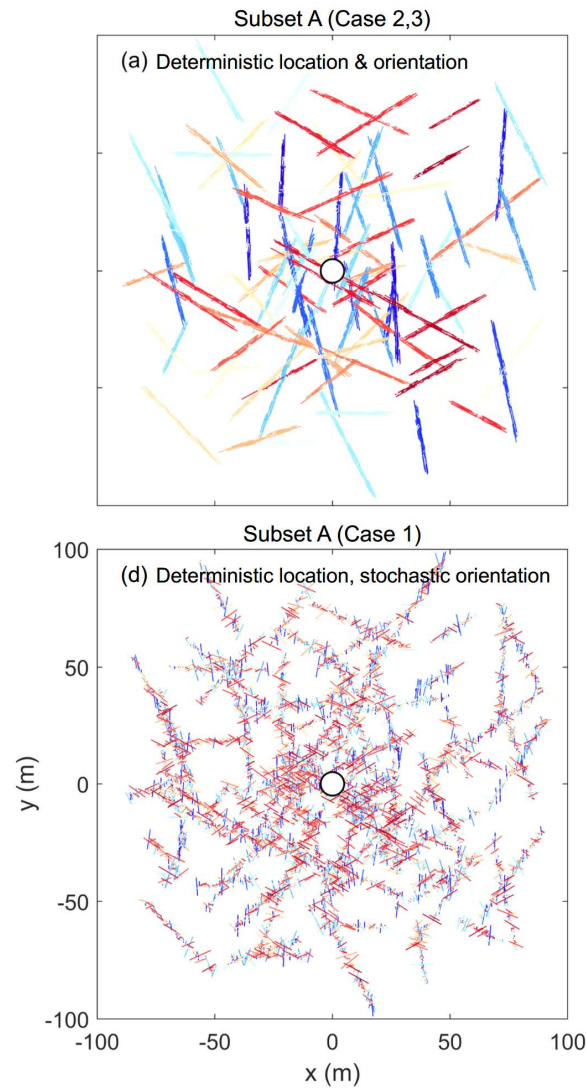

Subset B (Case 2,3)

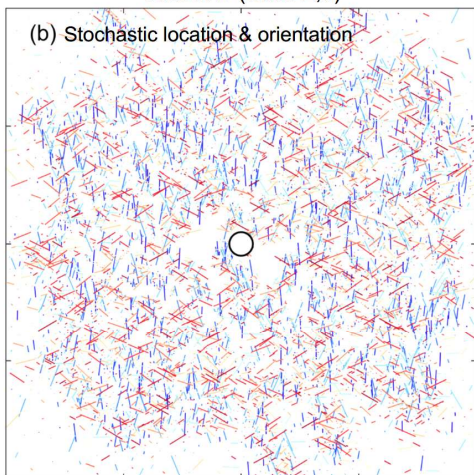

Subset B (Case 1)

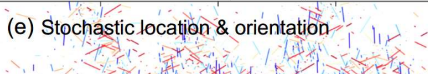

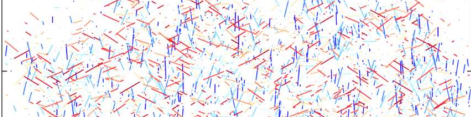
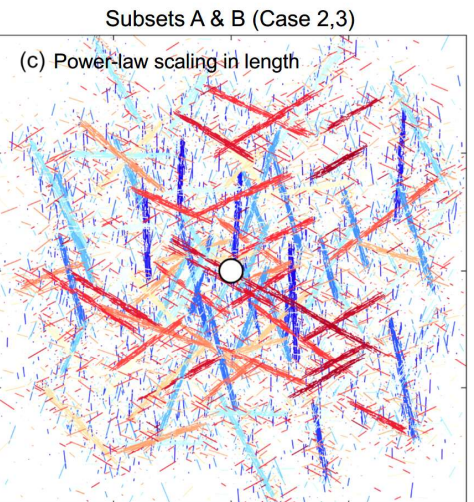

Subsets A \& B (Case 1)

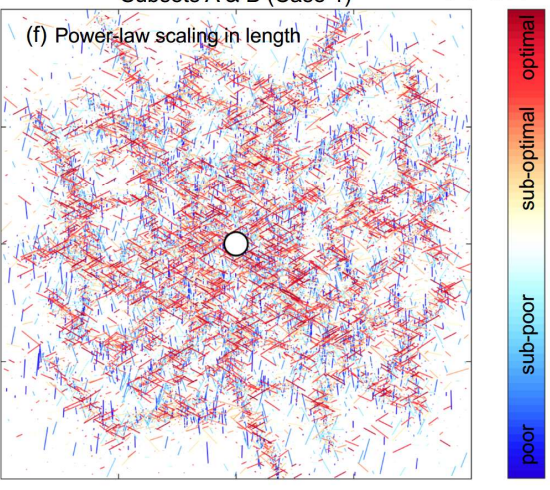

Figure 3. The dual fracture network (DF, equation (14)) consisting of 12800 fractures used for the seismicity modeling, shown together with its two subsets A and B. (a)-(c) Cases 2 and 3, and (d)-(f) case 1. Figures 3(a) shows the subset A with deterministic fracture locations and orientations as an approximation to the LSDF shown in Figure 2a; Figure 3(b) shows the subset $B$ as a stochastic realization of fractures in the hosting rock; Figure 3(c) shows the hybrid deterministicstochastic $D F$ in which the fracture length distribution follows a realistic power-law scaling relation. Figures $3 \mathrm{~d}-3 \mathrm{f}$ resemble Figures 3a-3c except for the stochastic fracture orientation in Figure 3d. In all figures, the warm color indicates the fracture is favorably oriented with respect to $\boldsymbol{\sigma}_{0}{ }^{\prime}$ whereas the cool color indicates otherwise.

357 For all cases, the same parameters are used: $\mu_{s}=0.6, \mu_{d}=0.4$ and $\boldsymbol{\sigma}_{0}{ }^{\prime}=[150 ; 05.05] \mathrm{MPa}$. Under the given $\boldsymbol{\sigma}_{0}{ }^{\prime}$, the initial effective normal stress and shear stress on all fractures are calculated, forming a Mohr circle, see Figure $4 \mathrm{a}$, where the color indicates the associated initial Coulomb stress $C F F_{0}$. The same color scale is used in Figure 3 to show the susceptibility of a fracture to slip with respect to $\boldsymbol{\sigma}_{0}{ }^{\prime}$. The peak and residual frictional strengths, calculated from $\mu_{s}$ and $\mu_{d}$, respectively, are also 362 shown in Figure 4a. Figure 4a also indicates that the domain is nearly critically stressed. Figure $4 \mathrm{~b}$ shows the distribution of $C F F_{0}$, which is no longer uniform, despite a uniform distribution in the fracture orientation. 


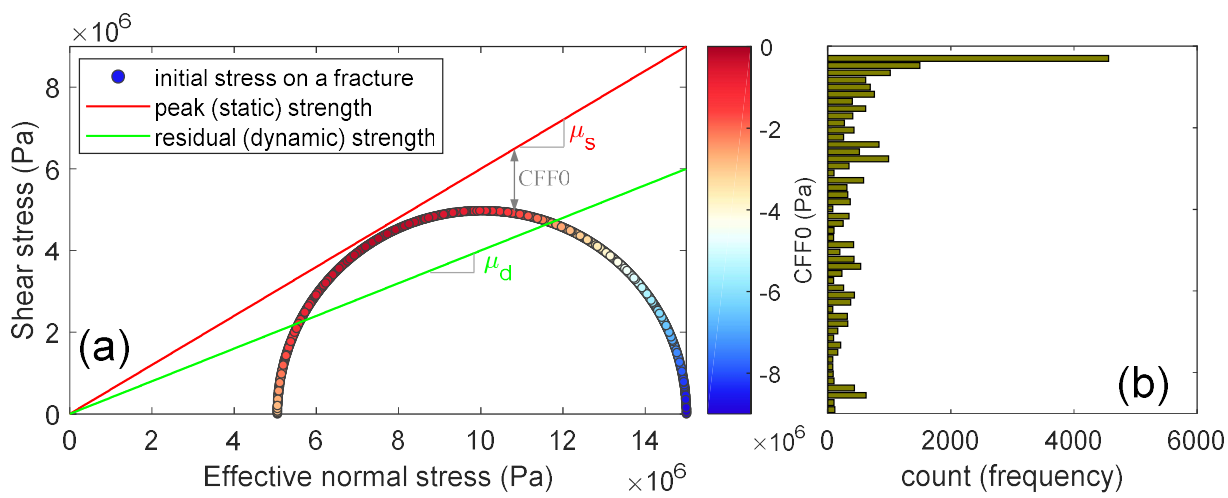

Figure 4. The initial stress used for the seismicity modeling. In Figure $4 \mathrm{a}$, the initial effective normal stress and shear stress on all fractures (Figures 3c, 3f) are plotted. Because the fractures uniformly sample all likely orientations, a Mohr circle is formed. The color indicates $C F F_{0}$. The peak and the residual strengths are also shown for reference (same as those in Figure 1). The geometric meaning of $C F F_{0}$ is shown for one fracture as an example. Figure $4 \mathrm{~b}$ is the histogram of $C F F_{0}$.

\section{Results}

\subsection{Fluid Pressure, Poroelastic Stress and Seismicity}

Figures 5 shows four snapshots of the distribution of $p$ (Figures 5a-5d) and the associated seismicity (Figures 5e-5h) for case 1. $p$ diffuses radially outward with a smooth and circular overpressure front (Shapiro et al., 1997), leading to a similar radially progressive distribution in the seismicity. However, this case has two differences from the diffusion-only statistical class of models (Shapiro et al., 2005). First, instead of using a predefined critical pore pressure value following a uniform distribution, we use predefined fractures with uniformly distributed orientations. Because the orientation needs to be transformed through equations (5)-(7), the resulting $C F F_{0}$ and the equivalent critical pore pressure, $\mu_{s} \times C F F_{0}$, follow a radically different distribution (Figure 4b), therefore, the seismicity distribution here is indeed different. Second, the use of predefined fractures further allows for the calculation of the seismic source parameters, including $M_{\mathrm{w}}$ and $\Delta \tau$ as are also shown in Figures 5e-5h. 


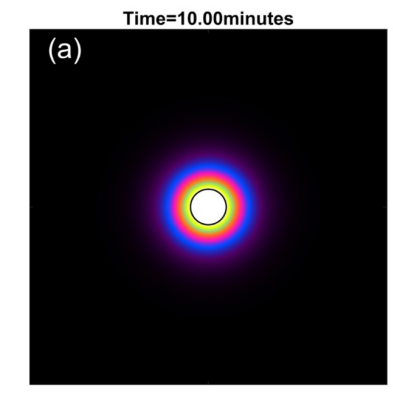

$$
\sqrt[50]{(e)}
$$

Time $=10.00$ minutes

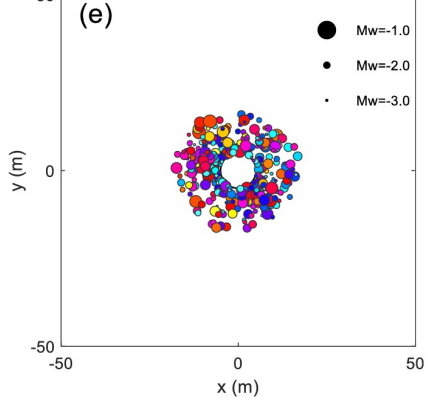

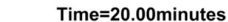

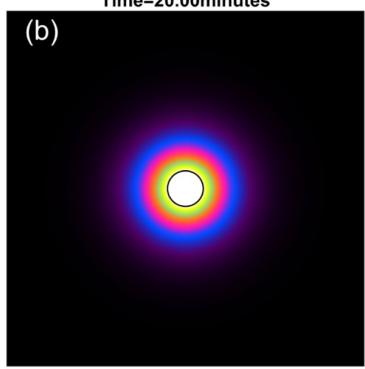

Time $=20.00$ minutes (f)

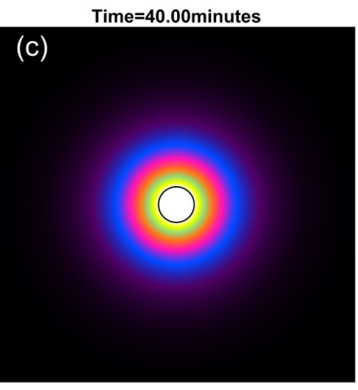

(g)

Time $=\mathbf{4 0} .00$ minutes

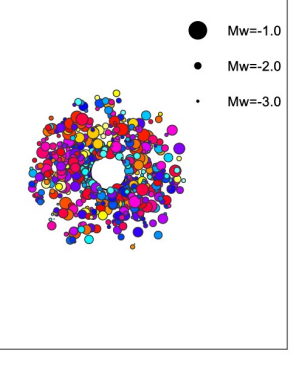

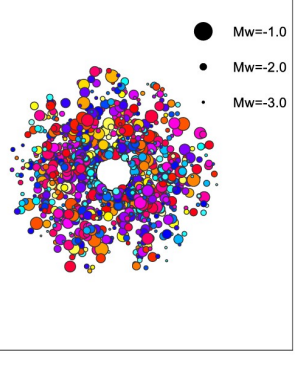

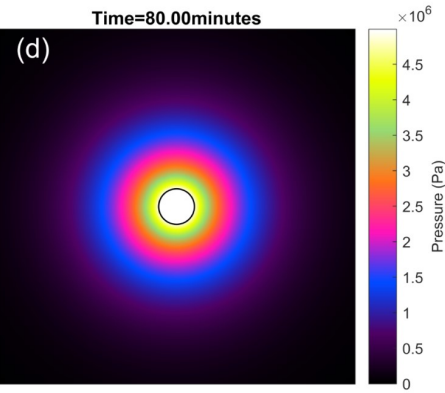

(h)

Time $=80.00$ minutes

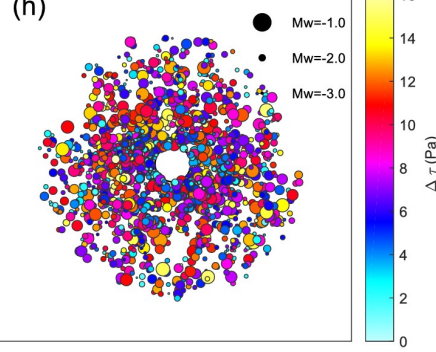

385

386

Figure 5. Snapshots of the spatial distribution of the modeled quantities at four time steps for case 1. (a)-(d) The fluid overpressure $p$ and (e)-(f) the seismicity sized with $M_{\mathrm{W}}$ and colored with $\Delta \tau$. Only the $100 \mathrm{~m} \times 100 \mathrm{~m}$ area around the center is shown. The time is indicated at the top of each plot.

Figure 6 shows the same snapshots of the same two quantities for case 2. Here, the effect of the LSDF (Figures 2a) becomes evident. First, $p$ increases primarily along those fractures and secondarily within the hosting rock, leading to a highly non-smooth overpressure front (Figures 6a-6d). Compared to case 1, $p$ here has a lower magnitude due to the LSDF diverting the fluid from the injector. Such a distribution leads to the clear clustering of the seismicity (Figures 6e-6h). Second, the distribution of the seismicity is not coincident with that of $p$; instead, the clustering occurs only along certain fractures. By further examining the fracture orientation (Figure 3a), we observe that the seismicity is clustered near those that are well-oriented or sub-well-oriented with respect to $\boldsymbol{\sigma}_{0}{ }^{\prime}$ and meanwhile subjected to sufficient $p$. 

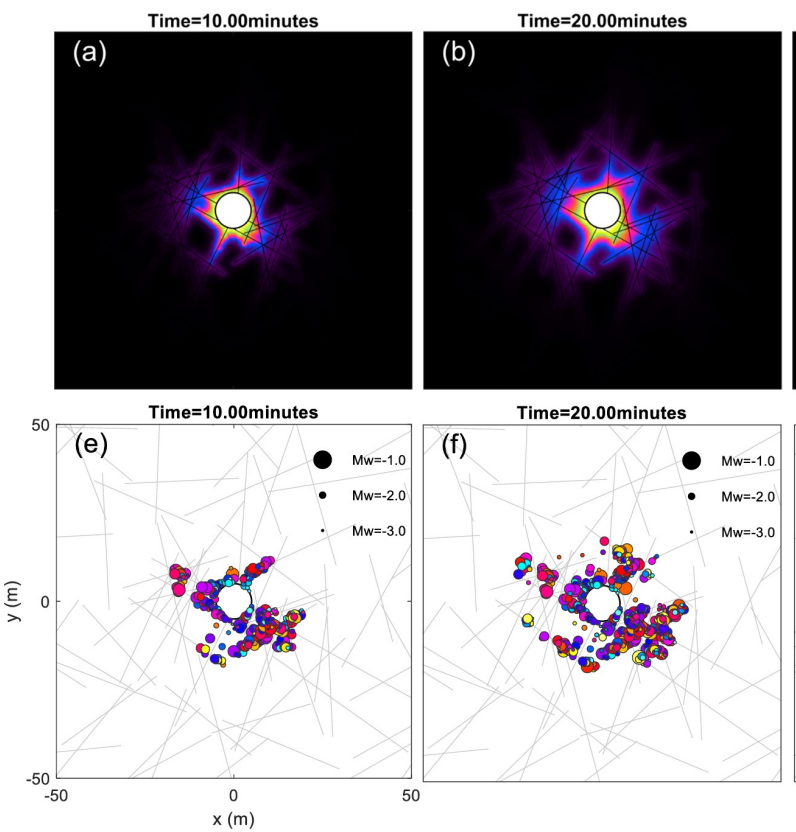

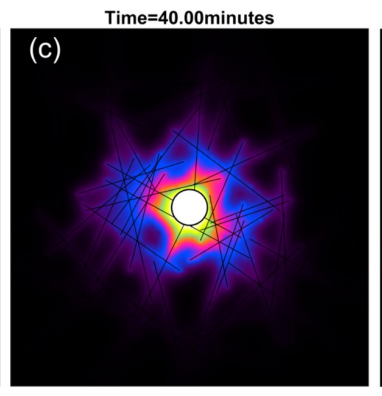

Time $=40.00$ minutes

(g)

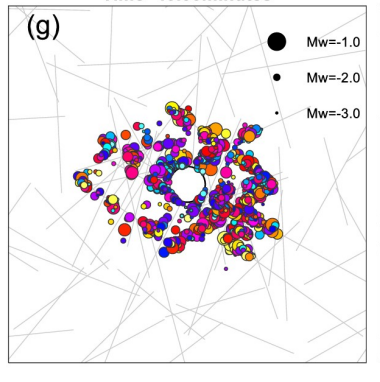

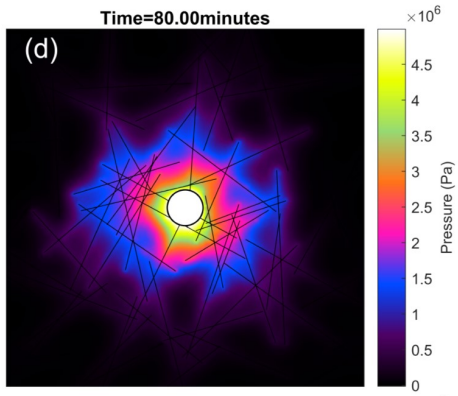

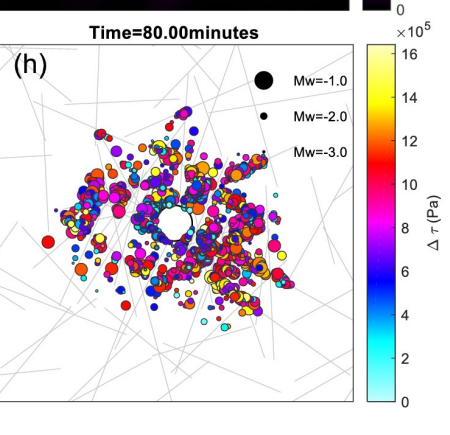

Figure 6. Same as Figure 5, but for case 2. The LSDF is shown in the background.

399 Figure 7 shows the results for case 3. The distribution of $p$ (Figures 7a-7d) and the seismicity 400 (Figures 7q-7t) are shown together with three other quantities, including (1) the first poroelastic stress invariant $I_{1}{ }^{\prime} / 3$ (Figures 7e-7h), (2) the second deviatoric poroelastic stress invariant $\sqrt{J_{2}{ }^{\prime}}$

402 (Figures 7i-7l) and (3) the excess poroelastic shear stress invariant $\sqrt{J_{2}{ }^{\prime}}-\sin (\phi) I_{1}{ }^{\prime} / 3$ (Figures $7 \mathrm{~m}$ 403 7p). All three quantities are calculated from $\boldsymbol{\sigma}_{p}{ }^{\prime}$ under plane strain (appendix A.4). Here, 404 compared to case 2, the effect of poroelastic coupling is shown. First, the distribution of $p$ is visibly different; the front of $p$ is suppressed and the magnitude becomes lower. Second, the poroelastic normal stress $I_{1}{ }^{\prime} / 3$ develops, dominantly being extensional near the fluid-penetrated fractures; however, the magnitude of $I_{1}{ }^{\prime} / 3$ is lower than that of its counterpart from the decoupled approach which predicts $I_{1}{ }^{\prime} / 3 \approx-0.67 p$ (see appendix A.4) using $p$ from case 2. Third, a pronounced shear stress field $\sqrt{J_{2}{ }^{\prime}}$ also develops and influences an even larger portion of the domain beyond the region subjected to $I_{1}{ }^{\prime} / 3$ and $p$, whereas its counterpart in case 2 is 0 . Fourth, as a result, the distribution of $\sqrt{J_{2}{ }^{\prime}}-\sin (\phi) I_{1}{ }^{\prime} / 3$ is different than its counterpart in case 2, which is

$4120.34 p$ (appendix A.4). Specifically, within the $p$ front (delineated in case 2, not case 3), the 413 magnitude is lower; outside the $p$ front, it still prevails. This observation has important 414 implications: within the fluid-pressurized region (i.e., in the near field), poroelastic coupling 415 tends to inhibit seismicity; outside this region (i.e., in the far field), it can either remotely promote 416 or inhibit seismicity depending on the fracture orientation. The reason behind the former is that 417 a fracture within the fluid-pressurized region acts as preferred flow channel, leading to a 418 discontinuous equivalent body force $(-\alpha \nabla p)$ acting away from it on the two sides, and therefore, 419 inhibiting shear mode failure by unclamping it (Chang \& Segall, 2016a; Jin \& Zoback, 2016b; Jin 420 \& Zoback, 2017). This is reflected by the modeled seismicity. Like in case 2, here the seismicity is 
421 clustered near fractures favorably oriented with respect to $\boldsymbol{\sigma}_{0}{ }^{\prime}$ and meanwhile subjected to 422 sufficient $\sqrt{J_{2}{ }^{\prime}}-\sin (\phi) I_{1}{ }^{\prime} / 3$. Notice the clustering is further enhanced by poroelastic coupling. More 423 importantly, the number of events in the near field is substantially reduced. In the far field, $\sqrt{J_{2}{ }^{\prime}}$ $424 \sin (\phi) I_{1}{ }^{\prime} / 3$ turns out to be minor and only a small number of events are remotely induced. Overall, 425 the event population is reduced to only around $1 / 3$ of that in case 2 . These observations are 426 further elaborated in section 4.3.
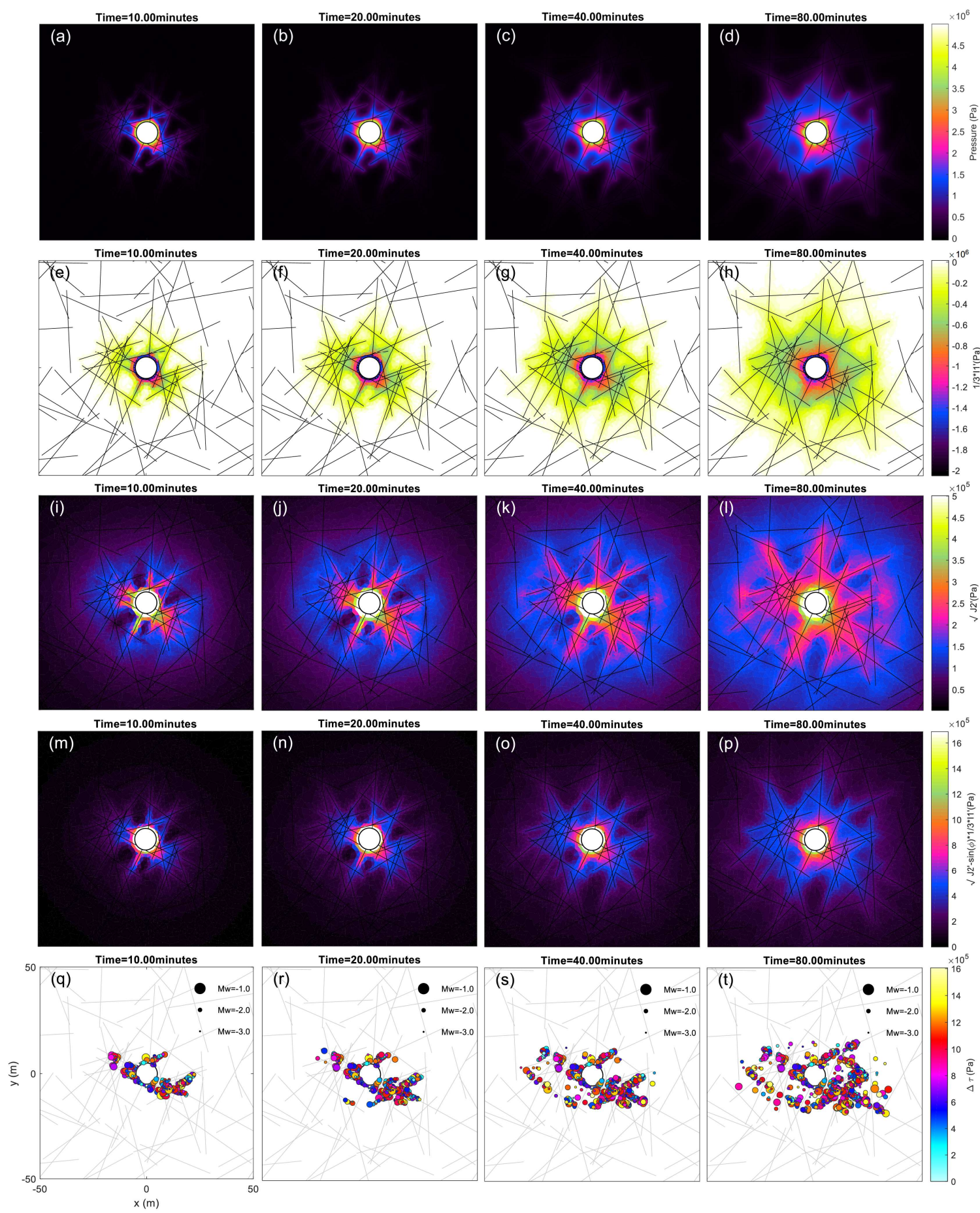
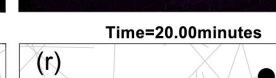

Time=20.00minutes

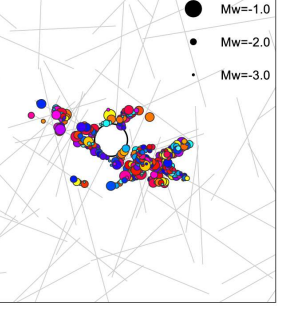

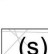

$(\mathrm{s})$
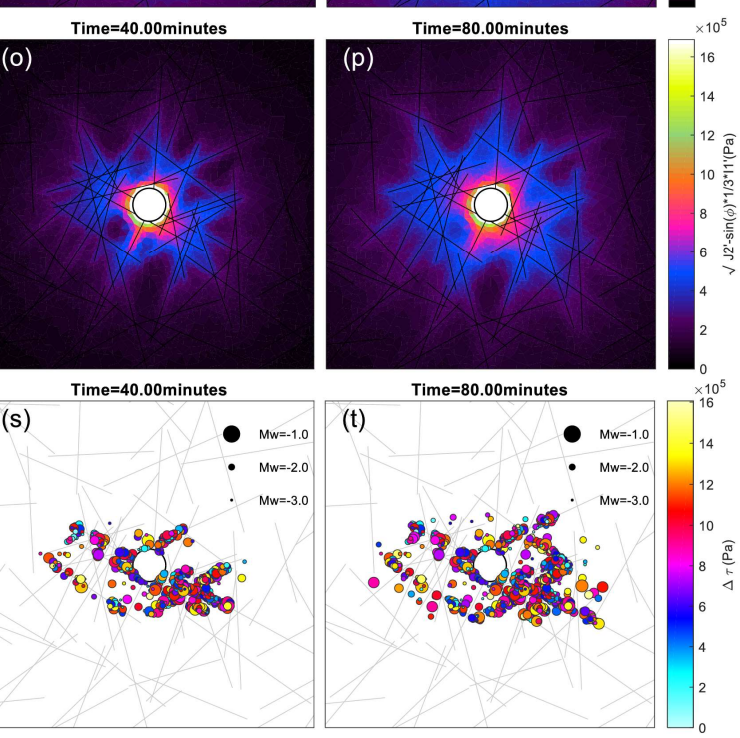

Time $=80.00$ minutes

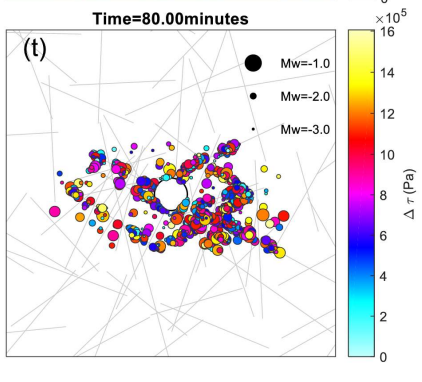


Figure 7. Snapshots of the spatial distribution of the modeled quantities at four time steps for case 3. (a)-(d) The fluid overpressure $p,(\mathrm{e})-(\mathrm{h})$ the first poroelastic stress invariant $I_{1}{ }^{\prime} / 3$, (i)-(l) the second deviatoric poroelastic stress invariant $\sqrt{J_{2}{ }^{\prime}},(\mathrm{m})-(\mathrm{p})$ the excess poroelastic shear stress invariant $\sqrt{J_{2}{ }^{\prime}}-\sin (\phi) I_{1}{ }^{\prime} / 3$ and $(\mathrm{q})-(\mathrm{t})$ the seismicity sized with $M_{\mathrm{w}}$ and colored with $\Delta \tau$. Only the $100 \mathrm{~m} \times 100 \mathrm{~m}$ area around the center is shown. The time is indicated at the top of each plot. The LSDF is shown in the background.

In Figures 5-7, the seismicity distribution shows increasing heterogeneity from cases 1 to 3 . The clustering of the events, as is frequently corroborated by field observations (e.g., Baisch \& Harjes, 2003; Stabile et al., 2014; Deichmann et al., 2014; Block et al., 2015), can only be modeled by resolving the LSDF. Additionally, we observe that the delineated seismicity front (Shapiro et al., $2005)$ is within the $p$ front in cases 1 and 2 and within the $\sqrt{J_{2}{ }^{\prime}}-\sin (\phi) I_{1}{ }^{\prime} / 3$ front in case 3 . This is because the domain is nearly critically stressed and even for the most optimally oriented fractures, a sufficient amount of $p$ or $\sqrt{J_{2}{ }^{\prime}}-\sin (\phi) I_{1}{ }^{\prime} / 3$ needs to be generated before triggering seismicity. We note here a 'front' is only used qualitatively and it refers to where changes in a quantity become visible. The modeling here highlights the importance of accounting for the interactions among fractures, the initial stress and poroelastic coupling.

\subsection{Event Classification}

Figure 8 shows the spatial-temporal evolution of all modeled events sized with $M_{\mathrm{w}}$ and colored with the event origin time $t_{0}$ for cases 1-3. The simulated duration of injection is 90 minutes. In addition to the spatial heterogeneity, the clustering and the event population reduction as explained in section 4.1, here the events also exhibit complex distribution in time for all cases. To better understand these events, we categorize them into different groups and compare the results among cases 1-3, as are shown through Figures 9 to 12.
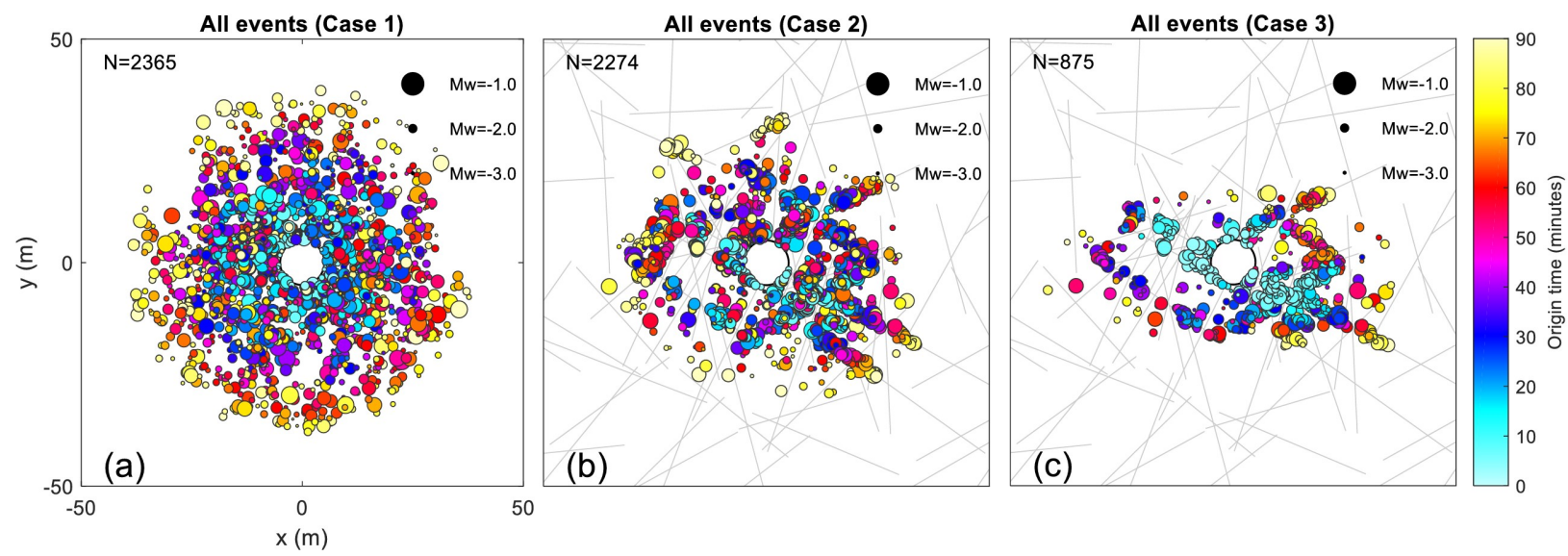

Figure 8. All events occurred within 90 minutes since the injection sized with $M_{\mathrm{w}}$ and colored with $t_{0}$. (a) Case 1 , (b) case 2 and (c) case 3 . Only the $100 \mathrm{~m} \times 100 \mathrm{~m}$ area around the center is shown. The number of events is indicated at the top left. The LSDF is shown in the background for cases 2 and 3. 


\subsubsection{Repeating Events}

Because we incorporated the poroelastic stress into seismic cycles, our model naturally produces repeating events, see Figure 9. Each location indicates a doublet pair or a multiplet group (e.g., Poupinet \& Ellsworth, 1984; Waldhauser \& Ellsworth, 2002) which contains two or more events that occur on the same source location but at different time; for visibility, a small-magnitude event is always plotted within a big-magnitude one (see the concentric circles). The repeating events exhibit some characteristics in space similar as those discussed in section 4.1. For example, the overall distribution is radial in case 1 but are clustered near favorably oriented fractures subjected to sufficient $p$ or $\sqrt{J_{2}{ }^{\prime}}-\sin (\phi) I_{1}{ }^{\prime} / 3$ in case 2 or 3 . In any case, they are concentrated in areas with a high event density. Further, despite the difference in the spatial pattern, the number of groups and the total number of events are similar between cases 1 and 2, suggesting the LSDF controls the distribution but probably not the population of the repeating events. In case 3, however, both drop significantly, suggesting poroelastic coupling inhibits the occurrence of repeating events as well in the near field. Finally, within each group, an earlier event does not necessarily have a larger magnitude; the contrary is not uncommon. This is due to the complex stress path (section 4.4) and the non-full degree of stress drop as is reflected by the $r$ in equation (18).
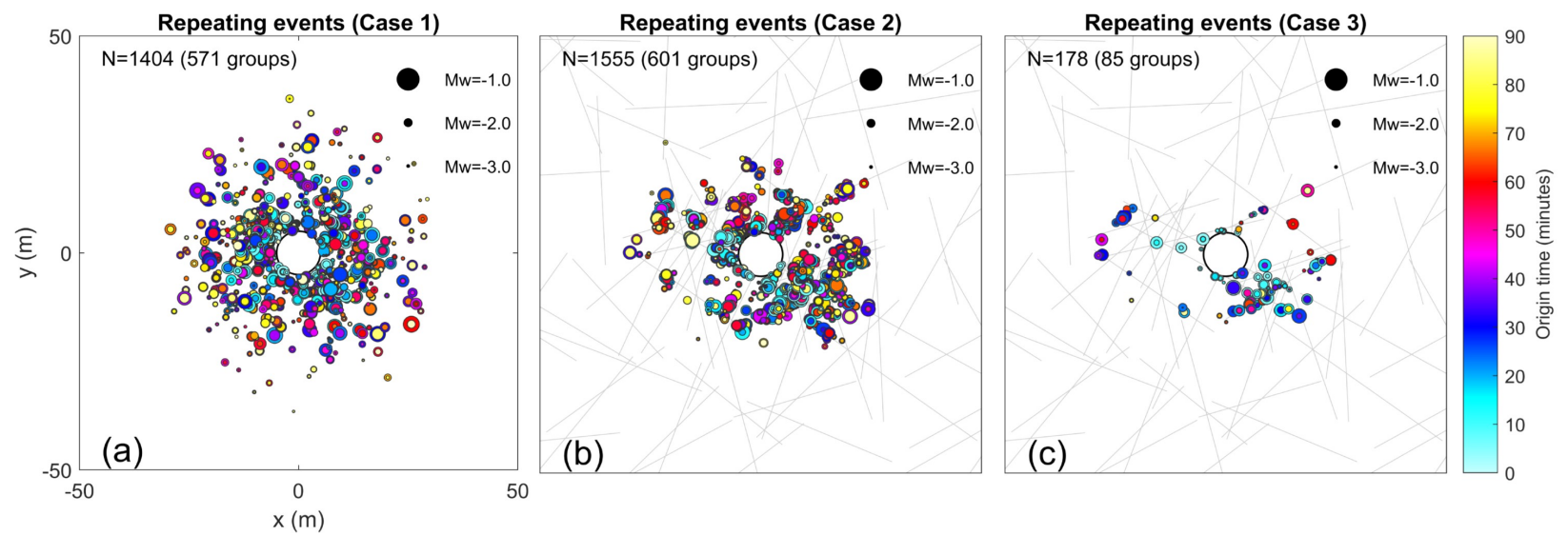

Figure 9. Repeating events sized with $M_{\mathrm{w}}$ and colored with $t_{0}$. (a) Case 1 , (b) case 2 and (c) case 3 . Only the $100 \mathrm{~m} \times 100$ $\mathrm{m}$ area around the center is shown. The number of groups and the total number of events are indicated at the top left. The LSDF in the background for cases 2 and 3.

To further understand the repeating events, we analyze the number of events within each group and the associated inter-seismic time, see Figure 10. From Figures 10a, 10c and 10e, one observes that in all cases, the repeating events are primarily doublet pairs; multiplet groups are present, and the number of events within these groups suggests that $p$ can drive a fracture through up to 8 seismic cycles within the simulated duration of injection; this number is reduced if poroelastic coupling is considered. For the entire catalog, the inter-seismic time between any two consecutive repeating events are compiled. The results are plotted in Figures 10b, 10d and 10f. The frequency drops approximately linearly with respect to the inter-seismic time for all cases and appears to be independent from fractures and poroelastic coupling. 

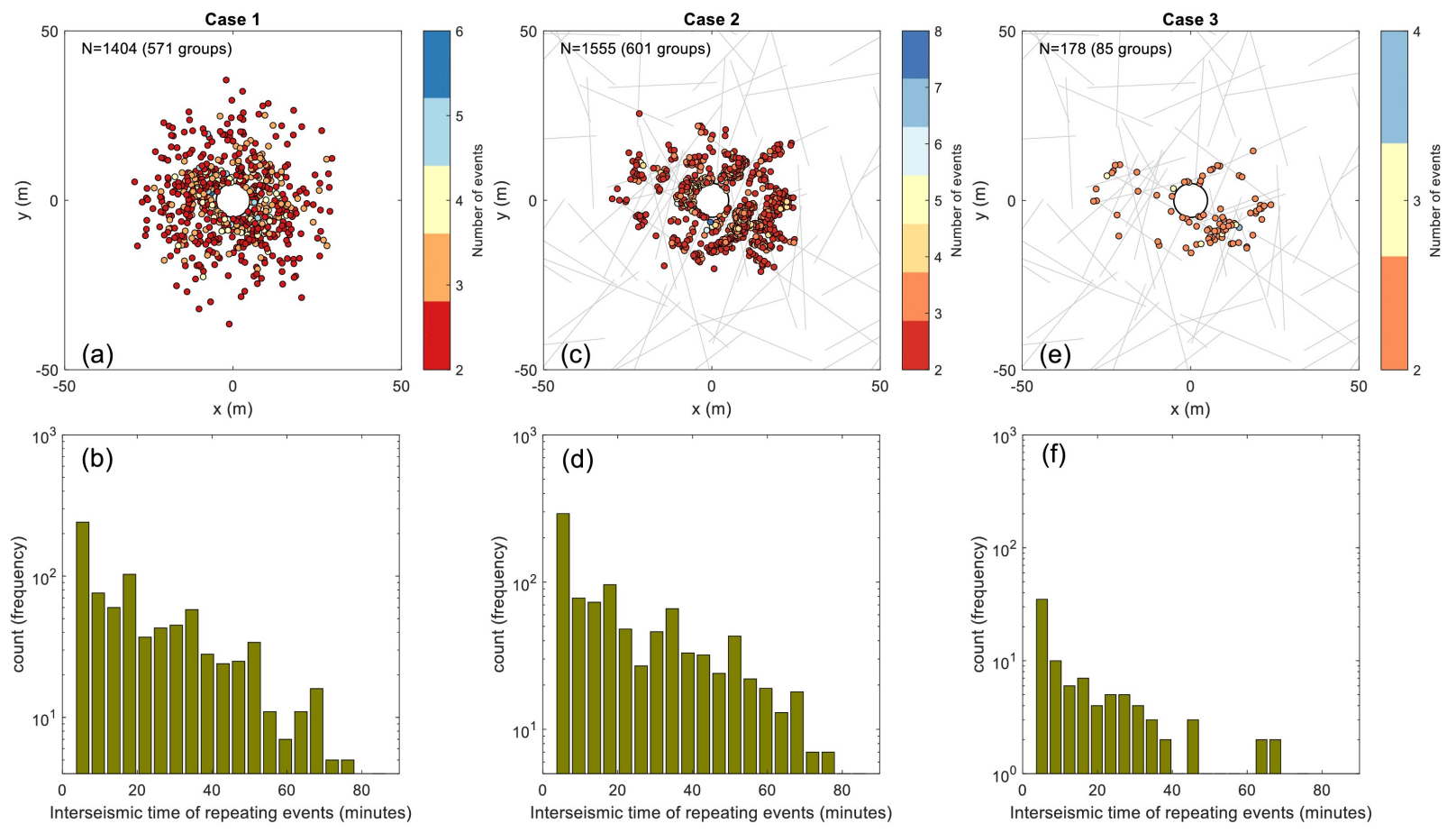

Figure 10. Characteristics of the repeating events. (a)-(b) Case 1, (c)-(d) case 2 and (e)-(f) case 3. Figures 10a, 10c and 10e show the location of each group containing repeating events, colored with the number of events within that group (i.e., the number of seismic cycles the associated fracture has undergone). Figures $10 \mathrm{~b}, 10 \mathrm{~d}$ and $10 \mathrm{f}$ are histograms showing the distribution of the inter-seismic time between two consecutive repeating events.

\subsubsection{Explicit and Implicit Events}

We also separate the events occurring along the LSDF (Figures 3a) from those within the hosting rock (Figures $3 b$ ), hereinafter referred to as the explicit and implicit events, respectively. Notice this classification should only apply to cases 2 and 3. The results are plotted in Figure 11. In both cases, the explicit events well depict lineation in alignment with the favorably-oriented deterministic fractures. The along-fracture distance of an explicit event correlates positively with its origin time. This is because for the same deterministic fracture, the orientation is identical and the required $p$ or $\sqrt{J_{2}{ }^{\prime}}-\sin (\phi) I_{1}{ }^{\prime} / 3$ is the same, therefore, the progressive increase in these two (see Figures 6 and 7) causes the seismicity to develop accordingly. For the implicit events, however, this trend immediately breaks down for the very same reason: the presence of the LSDF and the associated heterogeneity in $p$ or $\sqrt{J_{2}{ }^{\prime}}-\sin (\phi) I_{1}{ }^{\prime} / 3$, when acting on stochastic fractures of various orientations, lead to random spatial-temporal evolution of the seismicity within the hosting rock.

500 Additionally, poroelastic coupling seems to have the same effect on seismicity along deterministic fractures and within the hosting rock, as are indicated by the nearly $60 \%$ reduction in the population of both types of event. 

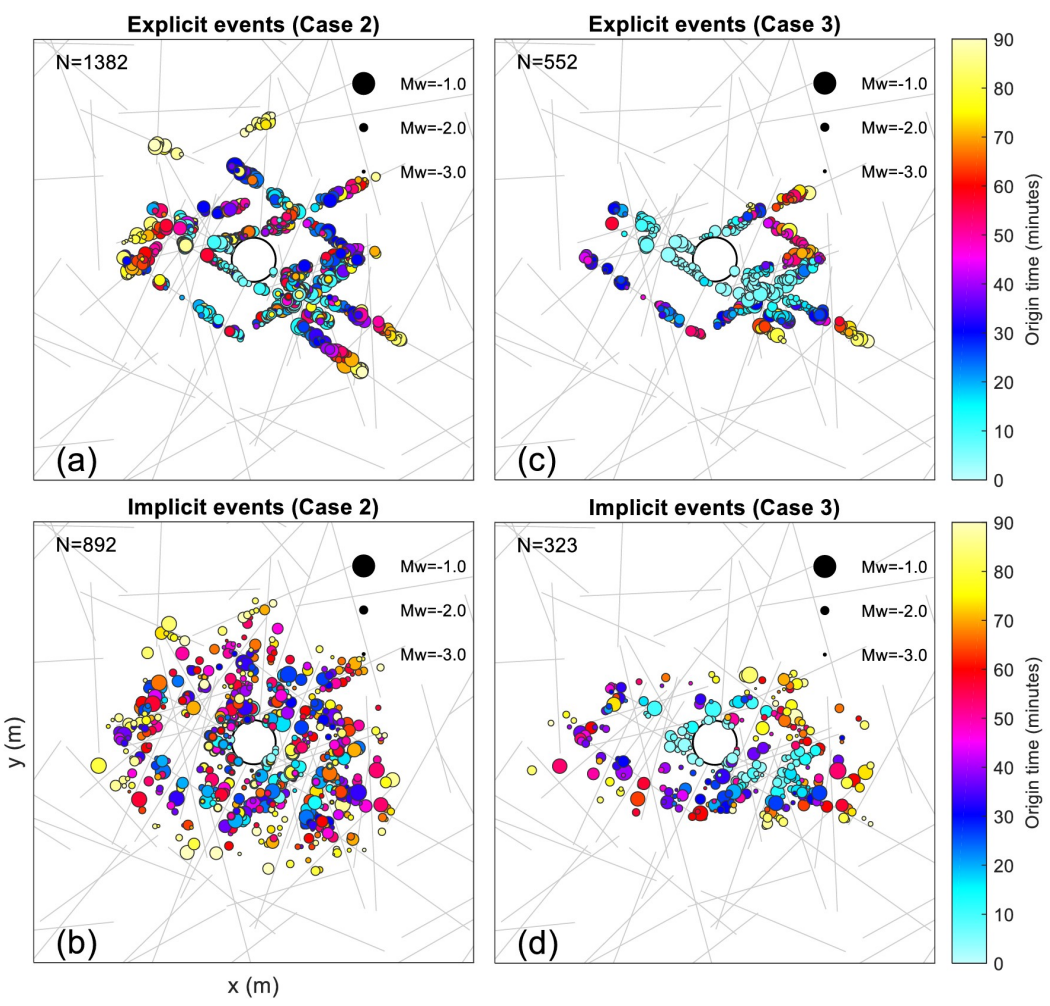

504 Figure 11. Explicit events (events along deterministic fractures) and implicit events (events within the hosting rock) sized with $M_{\mathrm{w}}$ and colored with $t_{0}$. (a)-(b) Case 2, (c)-(d) case 3. Only the $100 \mathrm{~m} \times 100 \mathrm{~m}$ area around the center is shown. The number of events is indicated at the top left. The LSDF is shown in the background.

\subsubsection{Triggered and Induced Events}

508 The triggered and induced events are distinguished from each other following the definition 509 proposed in appendix A.3 (see also Figure 1). The results are shown in Figure 12. In cases 1-3, $51093.3 \%, 92.8 \%$ and $98.5 \%$ of the events are triggered; the remaining small number of events are 511 induced and are distributed in close proximity to the injector, as they occur on unfavorably512 oriented fractures and require a significant amount of $p$ or $\sqrt{J_{2}{ }^{\prime}}-\sin (\phi) I_{1}{ }^{\prime} / 3$ to be activated. Again, 513 for either type of event, accounting for the LSDF leads to the clustering and accounting for 514 poroelastic coupling significantly reduces the number of events. 

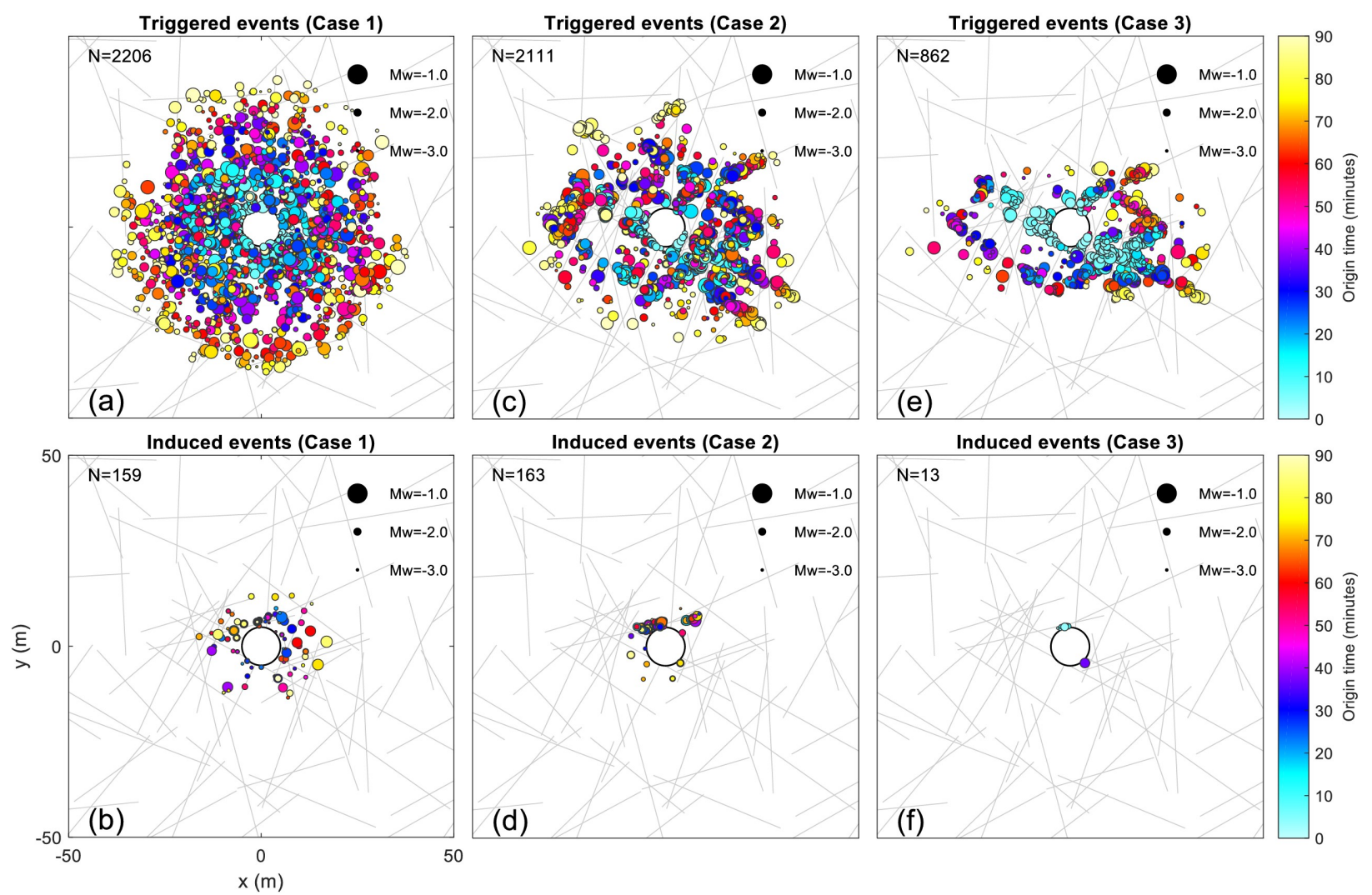

(c)
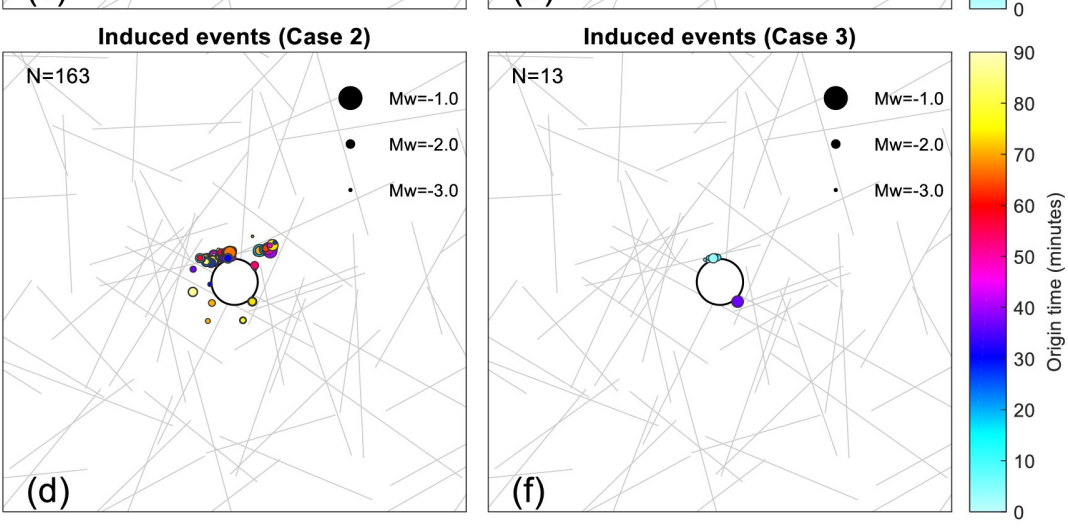

Figure 12. Triggered and induced events sized with $M_{\mathrm{w}}$ and colored with $t_{0}$. (a)-(b) Case 1, (c)-(d) case 2 and (e)-(f) case

3. Only the $100 \mathrm{~m} \times 100 \mathrm{~m}$ area around the center is shown. The number of events is indicated at the top left. The LSDF is shown in the background.

\subsection{R-T Characteristics}

\subsubsection{Fluid Pressure and Poroelastic Stress}

The spatial-temporal characteristics of the modeled quantities are further illustrated using the socalled $R-T$ plots shown in Figures 13-16, where $R$ is the distance from the origin and $T$ is the time since the beginning of the injection. $p$ is shown in Figure 13 for cases 1-3. Overlaying are several iso-diffusivity profiles (gray dashed lines) calculated as $R=\sqrt{4 \pi D_{h} T}+5 \mathrm{~m}$ where $D_{h}$ is the hydraulic diffusivity; $R=\sqrt{4 \pi D_{h} T}$ is a characteristic profile derived from a linear diffusion process resulting from a Heaviside point source injection in an isotropic, homogeneous and porous only medium, and is referred to as the so-called seismicity triggering front (Shapiro et al., 1997; Shapiro et al., 2002). Notice the use of such profiles should apply only to case 1 (Figure 13a). Nonetheless, for reference, they are also plotted for cases 2 and 3 (Figures 13b, 13c), where additionally, the green and magenta lines corresponding to $D_{h}$ of the hosting rock and the LSDF, respectively, are also plotted. It is mentioned in section 3.1 that in case $1 D_{h}=0.03 \mathrm{~m}^{2} / \mathrm{s}$. We choose this value such that the modeled $p$ front in the $R-T$ space is approximately the same as that in case 2 . In a sense, this value reflects the overall effective $D_{h}$ of the fractured porous media in case 2. Case 1 shows a smooth variation of $p$ in the $R-T$ space. In case 2 , however, due to the effect of fractures, strong 
heterogeneity is introduced, in addition to an overall reduction in the magnitude of $p$. The effect of poroelastic coupling is reflected by comparing case 2 and 3 . The $p$ front is slightly suppressed and the magnitude of $p$ is further reduced.
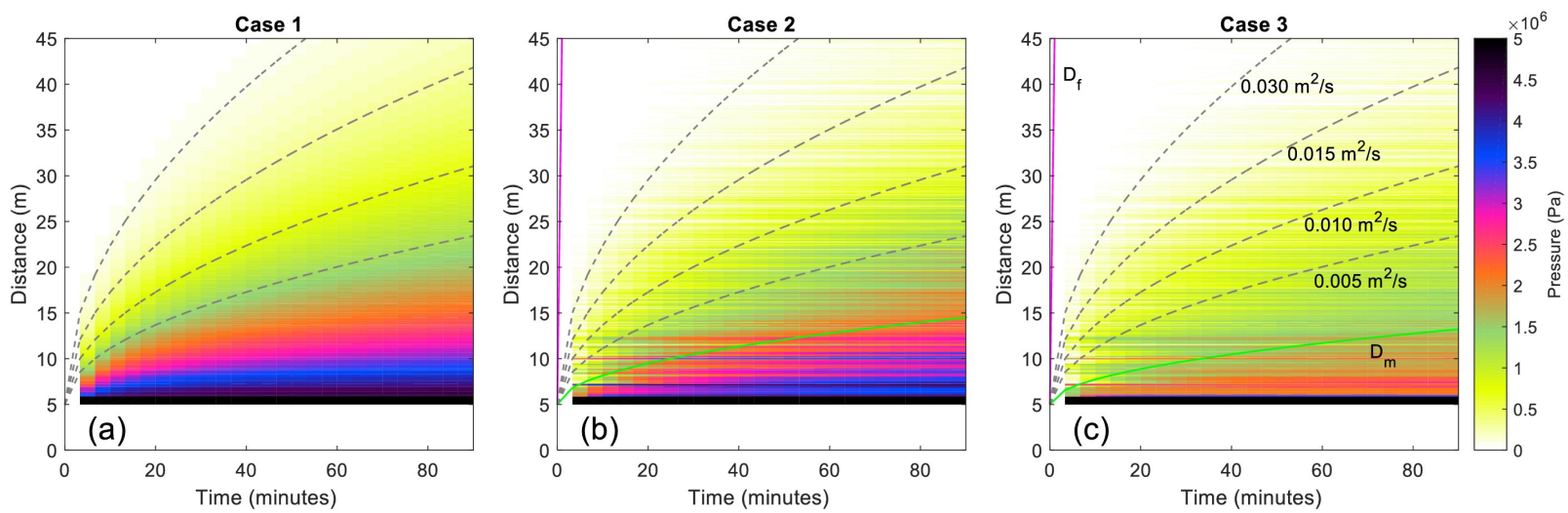

Figure 13. Space-time plot of the fluid overpressure $p$. (a) Case 1, (b) case 2 and (c) case 3. The distance is only plotted from 0 to $45 \mathrm{~m}$. The color scale is reserved from that in Figures 5-8. Several characteristic diffusion profiles are shown (see text) as references, including the green and magenta lines calculated using the diffussivity of the hosting rock and the fractures, respectively. The differnces between cases 1 and 2 show the effect of the LSDF and the differences between cases 2 and 3 show the effect of poroelastic coupling.

To further illustrate the effect of poroelasic coupling, for case 3 , we investigate the $R-T$ characteristics of the poroelastic stress invariants, see Figure 14. We observe the following. First, although the spatial distributions of $I_{1}{ }^{\prime} / 3$ and $p$ differ (Figures 7a-7h), the delineated front of $I_{1}{ }^{\prime} / 3$ (Figure 14a) coincides with that of $p$ (Figure 13c) in the $R-T$ space. This is explained by equation (A1) which states that $I_{1}{ }^{\prime} / 3$, which scales linearly with the volumetric strain $\nabla \cdot \underline{u}$, diffuses together with $p$. Poroelastic coupling does, however, reduces the magnitude of $I_{1}{ }^{\prime} / 3$ compared to its counterpart $-0.67 p$ (section 4.1 and appendix A.4) where $p$ is given by Figure 13b. The effect of poroelastic coupling is further manifested by Figure 14b, which shows the development of $\sqrt{J_{2}{ }^{\prime}}$ one-order below $p$ in magnitude. This cannot be predicted by case 2 . Also, it is evidently shown that the delineated front of $\sqrt{J_{2}{ }^{\prime}}$ well exceeds those of $p$ and $I_{1}{ }^{\prime} / 3$ (Figures 13c, 14a). Figure 14c results from the combination of Figures 14a and 14b. The effect of poroelastic coupling is reflected by its difference in magnitude from its counterpart $0.34 p$ (section 4.1 and appendix A.4) where $p$ again is given by Figure 13b. Finally, poroelastic coupling also seems to smear out the heterogeneity in the stress upon comparing Figures 14a-14c against Figure 13b. Notice equation (A6) is not included in our modeling. 

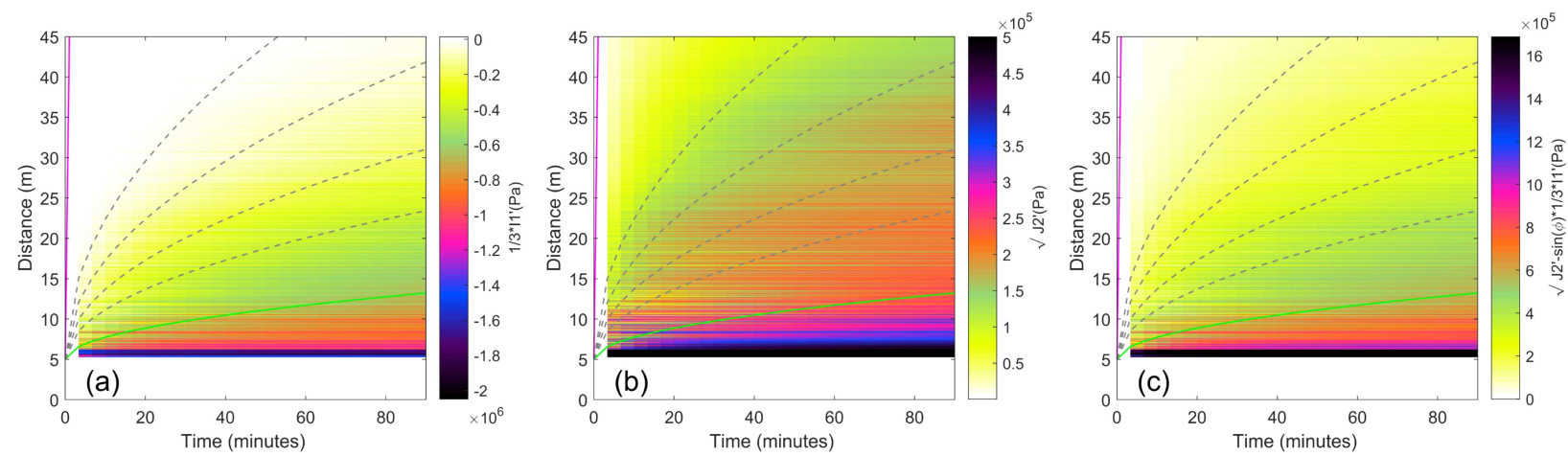

560

561

562

563

564

565

566

567

568

569

570

571

572

573

574

575

576

577

578

579

580

581

582

583

584

585

586

587

588

Figure 14. Space-time plot of the poroelastic stress invariants for case 3. (a) $I_{1}{ }^{\prime} / 3$, (b) $\sqrt{J_{2}{ }^{\prime}}$ and $\left(\right.$ c) $\sqrt{J_{2}{ }^{\prime}}-\sin (\phi) I_{1}{ }^{\prime} / 3$. The distance is only plotted from 0 to $45 \mathrm{~m}$ and the characteristic diffusion profiles are the same as those in Figure 13 . The color scale is reserved from that in Figures 5-8. The counterparts of the three quantities in case 2 without the coupling effect can be obtained by multiplying the $p$ in Figure $13 \mathrm{~b}$ with $-0.67,0$ and 0.34 (appendix A.4).

\subsubsection{Seismicity}

Figures 15 shows the $R-T$ distribution of the seismicity for cases 1-3 and the color indicates $C F F_{0}$. In Figure 15a, a parabolic seismicity front is clearly delineated for case 1, showing also an evident 'lag' behind the $p$ front (Figure 13a). This lag reflects the effect of the initials stress with respect to the static shear failure line (i.e., the peak strength, see Figure 4). Here $D_{h}$ corresponding to the $p$ front and the seismicity front are $0.03 \mathrm{~m}^{2} / \mathrm{s}$ and $0.015 \mathrm{~m}^{2} / \mathrm{s}$, respectively. In this case, if the seismicity front was to be used to back calculate $D_{h}$ (e.g., Shapiro et al., 2002), $D_{h}$ would be overestimated by $100 \%$. This motivates some nonlinear diffusion-based interpretations which incorporate pressure-dependent $D_{h}$ (e.g., Hummel \& Shapiro, 2012; Hummel \& Shapiro, 2013). Here, our model is mechanics-based and it does not require the somewhat unclear definition of 'a relatively large $p$ ' which underlines the diffusion-only class of statistical models (Shapiro et al., 1997). The effect of the LSDF can be seen in Figure 15b. Notice the increased curvature of the parabolic seismicity front, which is above the predicted characteristic profile (second grey dashed line from the top) earlier and near the injector but below this profile later and away from the injector. Hummel \& Shapiro (2013) used a power-law type of pressure-dependent $D_{h}$ to correct for this change. However, our model not only produces this change but also introduces additional heterogeneity. Figure 15c shows further variations by accounting for poroelastic coupling. Compared to Figure 15b, here the number of events is greatly reduced, the heterogeneity becomes much more pronounced, and some 'outliers', which are the remotely triggered events, are present but not dominant. Additionally, nearly all events are sourced from favorably-oriented fractures. The result of case 3 also shows a good agreement with a dataset provided in Hummel \& Shapiro (2013). Finally, the same $R$ - $T$ plots are made using only the repeating events for cases $1-3$, as are shown in Figures 15d-15f, which illustrate the 'breaking-down' of the parabolic seismicity front for repeating events. Such events are assumed to be non-existent in the diffusion-only class of statistical models. 

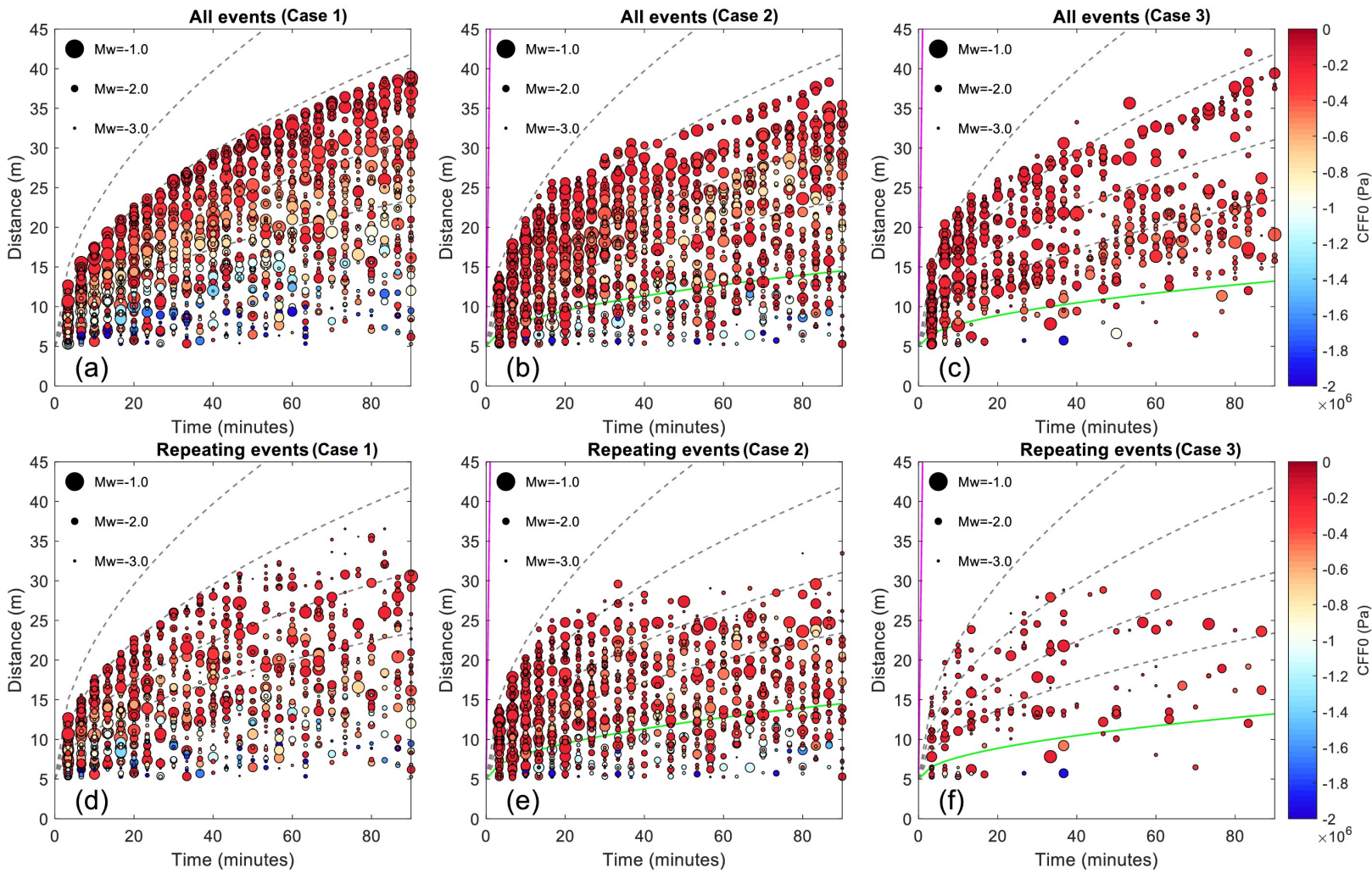

Figure 15. Space-time plot of all seismic events and repeating seismic events, sized with $M_{\mathrm{w}}$ and the colored with $C F F_{0}$, (a), (d) Case 1, (b),(e) case 2 and (c),(f) case 3. The distance is only plotted from 0 to $45 \mathrm{~m}$ and the reference characteristic diffusion profiles are the same as those in Figure 13. The differnces between cases 1 and 2 show the effect of the LSDF and the differences between cases 2 and 3 show the effect of poroelastic coupling.

\section{$594 \quad 4.4$ Stress History}

595 As an example, for each case, we chose one representative fracture that has generated the most repeating events and plot the associated complete stress path colored with time, see Figure 17. In all cases, $p$ or $\sqrt{J_{2}{ }^{\prime}}-\sin (\phi) I_{1}{ }^{\prime} / 3$ are sufficient enough to drive a fracture through multiple seismic cycles within 90 minutes. However, the decoupled approach tends to over-predict the number of 599 seismic cycles (see also Figure 10). Notice the increasingly unfavorable orientation of the fracture 600 from cases 3 to 1 . Additionally, within each seismic cycle, poroelastic coupling leads to a bended stress path in case 3 as opposed to a linear leftward one in case 1 or 2.
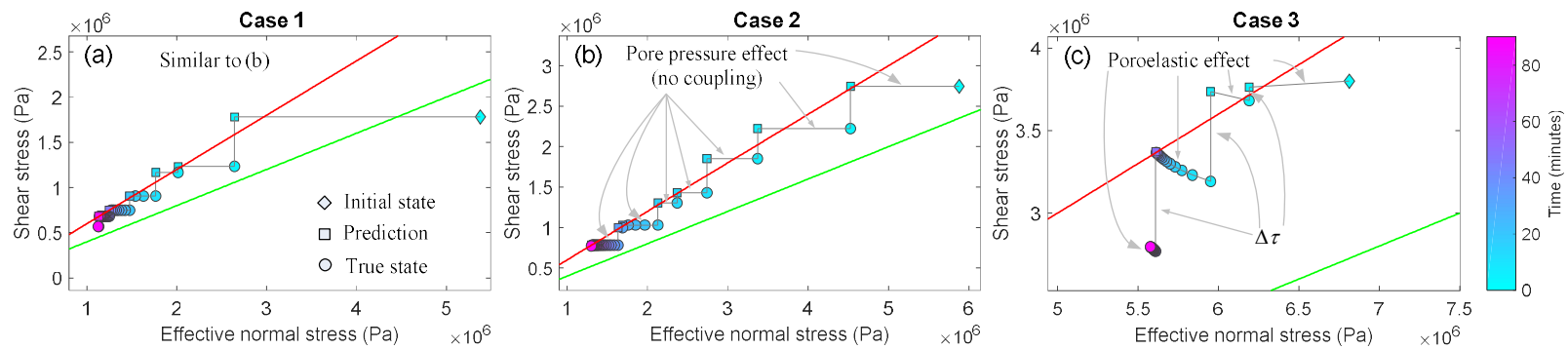
Figure 16. Representative complete stress paths. (a) Case 1, (b) case 2 and (c) case 3. The color indicates the time. The number of seismic cycles is 6 in cases 1 and 2 and 3 in case 3 . The pore pressure effect and the poroelastic effect are indicated.

Figure 17 gives the snapshots of changes in the stress of all fractures (Figures 3c, 3f) in the $\sigma_{n}{ }^{\prime}-\tau$ space for cases 1-3. We hereinafter abbreviate each $\sigma_{n}{ }^{\prime}-\tau$ pair as a $N S$, which is indicative of a fracture. The reference state (Figure 4a) is divided into three parts, namely parts I, II and III. Upon injection, the stress state on some fractures deviates from the reference state, and the relative changes are shown by the grey arrows. Cases 1 (Figures 16a-16d) assumes simply the pore pressure effect. As a result, $p$ always causes a reduction in $\sigma_{n}{ }^{\prime}$ by the amount of $\alpha p$ but does not change $\tau$, leading to a strict leftward translation of a NS before it reaches the peak strength and $C F F$ remains negative. When CFF reaches 0 , seismicity occurs and $\Delta \tau$ is enforced. Throughout this process, a NS must remain constrained below the peak strength at all time, and if seismicity occurs, above the residual strength. This means a NS originated from part II remains in between the green line and the red line, and a NS from part I can cross the green line if $p$ is sufficient but always stays below the red line; correspondingly, the triangular domains denoted as B and A (dashed lines) define the respective possible new stress state of a fracture driven to failure from parts II and I. Therefore, the pore pressure effect also predicts a positive correlation between the favorability of the orientation and $\Delta \tau_{\text {max }}$. Here, any arrow with a downward component signifies the seismicity only. As can be seen, the majority of the events are sourced from part II. For part III, a similar triangular domain $\mathrm{C}$ can be defined. All the above observations hold for case 2 (Figures 16e-16h). However, compared to case 1, here the deviation of a NS is more discernable from others due to the localization of $p$ around the LSDF. The magnitude of $p$ in general becomes lower as is reflected by the less amount of leftward translation. The results of case 3 (Figures 16i161) show the intriguing effect of poroelastic coupling. The deviation from the Mohr circle is much less significant in general and the seismicity is inhibited overall. Notice the deviation of a NS is now towards all directions, suggesting any combination of an increase or decrease in $\sigma_{n}{ }^{\prime}$ and an increase or decrease in $\tau$ is possible. For example, a NS from part I can undergo a left and upward path towards the peak strength, rendering a larger possible $\Delta \tau_{\max }$. As a result, domains $\mathrm{A}, \mathrm{B}$ and $\mathrm{C}$ can no longer be defined here. An arrow with a downward component indicates either the seismicity or the poroelastic shear stress. Nonetheless, for a majority of the fractures and prior to the seismicity, the leftward component still dominates over the others, suggesting the reduction in $\sigma_{n}{ }^{\prime}$ is the primary source driving up CFF. 


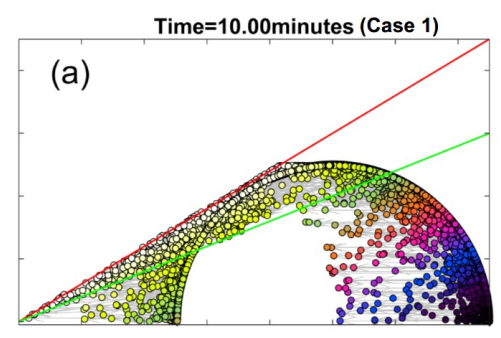

Time $=\mathbf{2 0}$.00minutes $($ Case 1 )

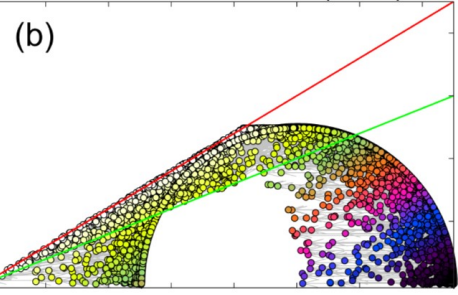

Time $=40.00$ minutes $($ Case 1$)$

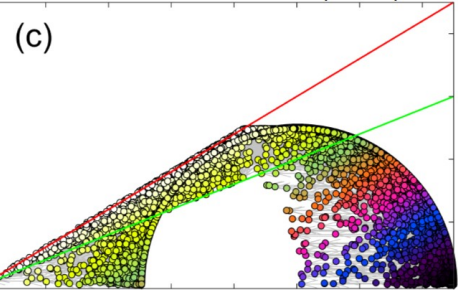

Time $=80.00$ minutes $($ Case 1$)$

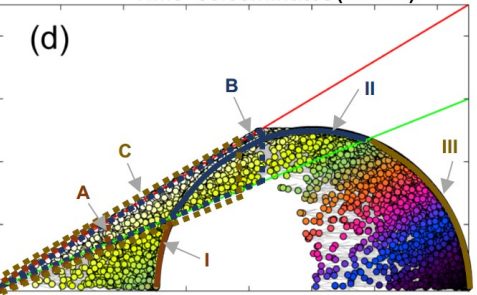

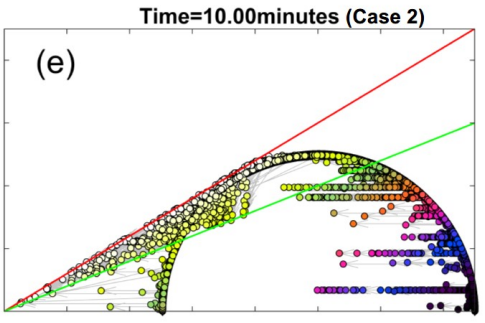

Time $=\mathbf{2 0 . 0 0 m i n u t e s}$ (Case 2)

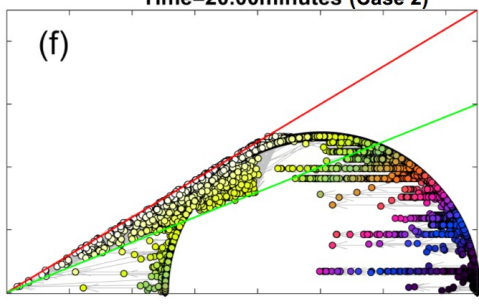

Time=40.00minutes (Case 2)

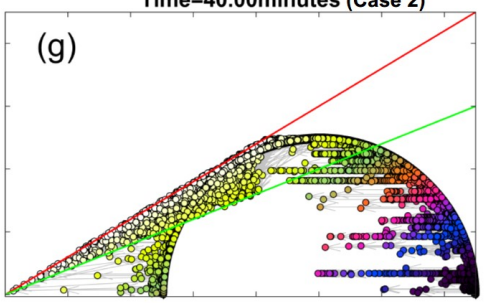

Time $=80.00$ minutes $($ Case 2$)$

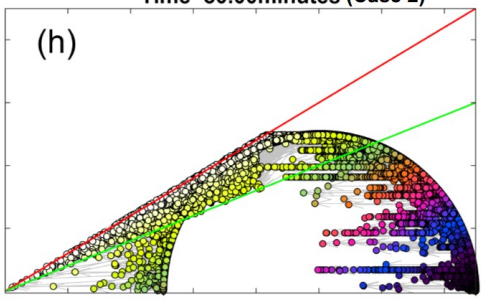

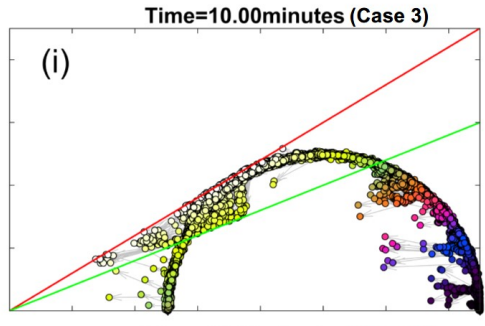

Time $=\mathbf{2 0 . 0 0 m i n u t e s}$ (Case 3 )

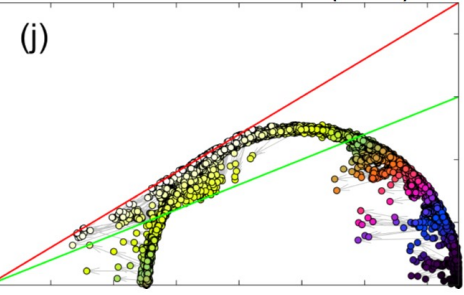

Time $=40.00$ minutes $($ Case 3 )

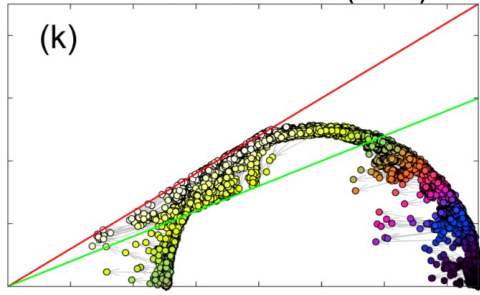

$\times 10^{6} \quad$ Time $=80.00$ minutes $($ Case 3$)$

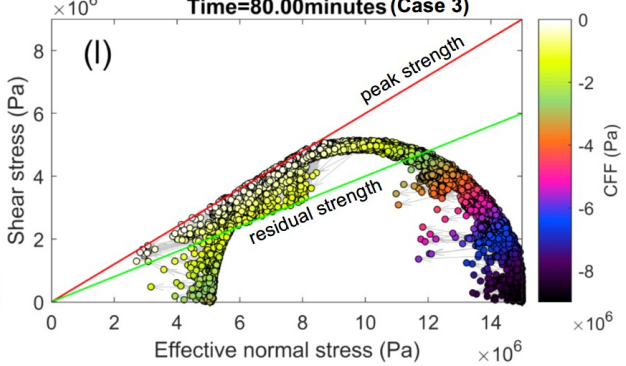

Figure 17. Snapshots of the effective normal stress and shear stress on all fractures showing the deviation from the initial reference state (the Mohr circle in Figure 4a) at four selected time steps. (a)-(d) Case 1, (e)-(h) case 2 and (i)-(l) case 3. The peak and residual strengths are shown for reference. The color indicates CFF and the time is indicated at the top of each plot. For each fracture, two dots corresponding the initial and new stress states are plotted, connected with an arrow indicating the relative change. The initial Mohr circle is partitioned into three parts labeled as I, II and III. The meaning of the triangular areas bounded with dashed lines are explained in the text. The differnces between cases 1 and 2 show the effect of the LSDF and the differences between cases 2 and 3 show the effect of poroelastic coupling.

\section{$644 \quad 4.5$ Source Parameters}

\subsubsection{Stress Drop, Fracture Length and Moment Magnitude}

646 Figures 18a, 18c and 18e summarize the modeled seismic source characteristics in the parameter 647 space for cases 1-3. For each event, $M_{\mathrm{w}}$ is plotted against the associated fracture length $L$ and 648 colored with $\Delta \tau$. The modeled events, with $M_{\mathrm{w}}$ between -3 and -1 , occur on fractures of $L$ ranging 649 from $0.1 \mathrm{~m}$ and $10 \mathrm{~m}$, and $\Delta \tau$ ranges from below $0.1 \mathrm{MPa}$ to above $1 \mathrm{MPa}$, consistent with many 650 real micro-earthquake data sets (e.g., Goertz-Allmann et al., 2011; Mukuhira, 2013). Such source 
characteristics overall seem not affected by the LSDF nor poroelastic coupling. For a realistic range of $\Delta \tau$, the parameter $r$ in equation (18) turns out to be important, see appendix A.6. Figures $18 \mathrm{~b}, 18 \mathrm{~d}$ and $18 \mathrm{f}$ further show the overall similar distribution of $\Delta \tau$ for cases $1-3$. In each case, [0.1, 1] $\mathrm{MPa}$ is the dominant range. In case 3 , however, events with high $\Delta \tau$ (e.g., above $1 \mathrm{MPa}$ ) does occupy a higher percentage, consistent with that poroelastic coupling can lead to a larger possible $\Delta \tau_{\max }$ as demonstrated in section 4.4.
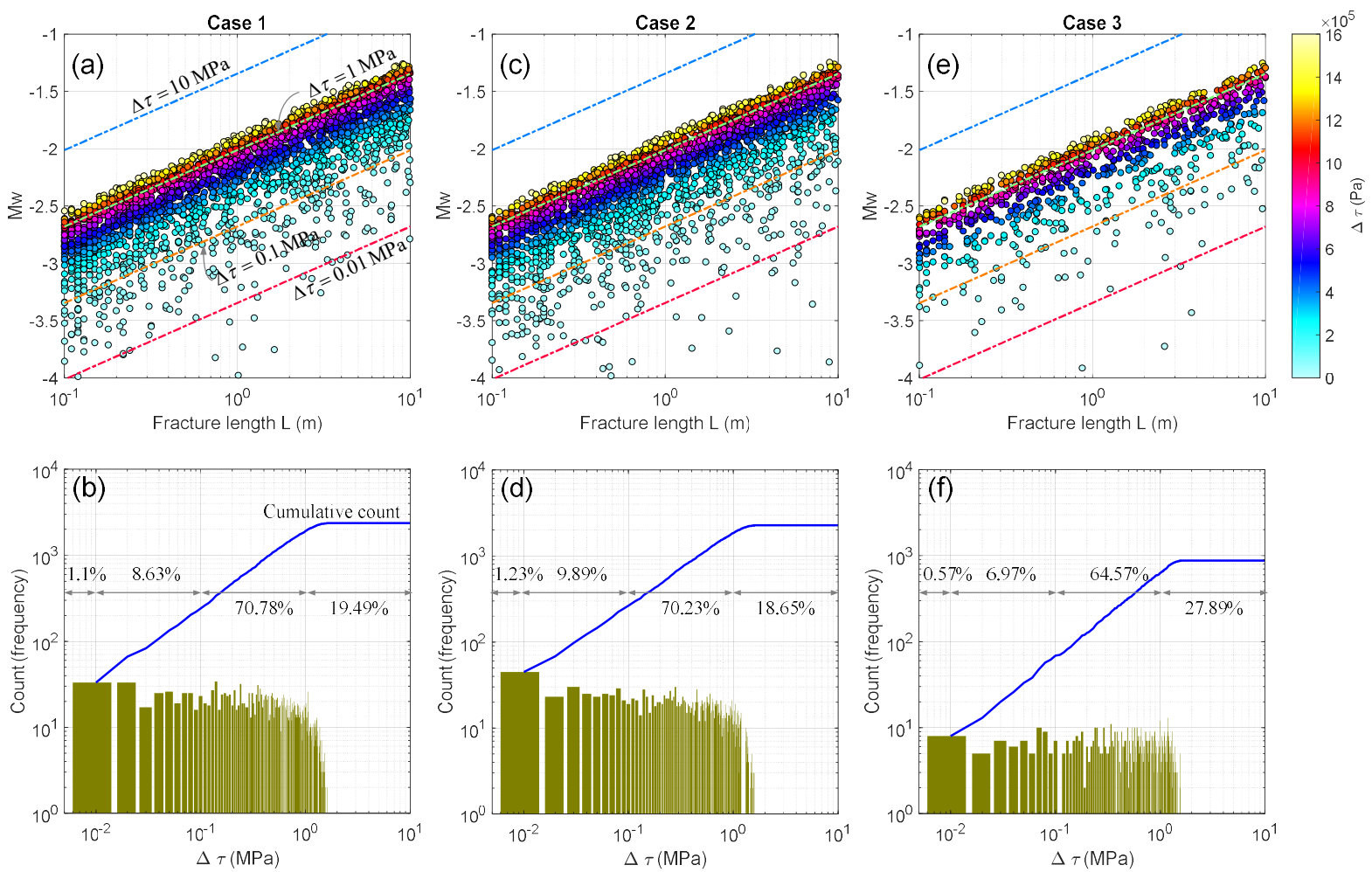

Figure 18. The top row shows relationships among $M_{\mathrm{w}}, L$ and $\Delta \tau$ of all modeled events. Overlaying are four contours corresponding to $\Delta \tau=0.01 \mathrm{MPa}, 0.1 \mathrm{MPa}, 1 \mathrm{MPa}$ and $10 \mathrm{MPa}$. The bottom row shows the histograms of $\Delta \tau$ together with the cumulative frequency using 1000 equal-sized bins on the range $[0.01,10] \mathrm{MPa}$. Additionally, the number of events with $\Delta \tau \leq 0.01 \mathrm{MPa}, 0.01 \mathrm{MPa}<\Delta \tau \leq 0.1 \mathrm{MPa}, 0.1 \mathrm{MPa}<\Delta \tau \leq 1 \mathrm{MPa}$ and $\Delta \tau>1 \mathrm{MPa}$ are counted and the percentages are shown. (a), (b) Case 1, (c), (d) case 2 and (e), (f) case 3.

\subsubsection{Magnitude-Frequency Relation}

We have introduced a power law that describes the commonly observed scaling relation between the fracture length and the frequency (section 3.2). On the other hand, earthquakes in nature are characterized with a universal statistical relation between the magnitude and the cumulative frequency, namely the Gutenburg-Richter law (Gutenberg, 1956), which reads:

$$
\lg N\left(m>M_{w}\right)=a-b M_{w}
$$

where $N\left(m>M_{\mathrm{w}}\right)$ is the total number of events with a moment magnitude $m$ above $M_{\mathrm{w}}$, and $a$ and $b$ are constants. 
In nature, $D$ is frequently observed to be between 1 and 2 (e.g., Okubo \& Aki, 1987), whereas a common value of $b$ is around 1 (e.g., Shi \& Bolt, 1982). Studies suggest that $D$ and $b$ are inherently related. For example, Hirata (1989) suggests that $D \approx 2 b$. What is somewhat curious is that for induced seismic events, $b$ is often above 1 (e.g., Vermylen \& Zoback, 2011) and sometimes around 2 (e.g., Bachmann et al., 2012), although a near-1 value has also been reported (Schoenball et al., 676 2015).

In Figure 19, for each case, the distribution of the length of all fractures (Figures 3c, 3f) is plotted (green), together with the power law fitting line (magenta); the distribution of the length of the activated subset of fractures is also plotted (red), which clearly no longer obeys the power law decay, owing to that only favorably oriented fractures are induced to slip. Nonetheless, the magnitude-frequency relation still holds for the induced events, as is illustrated in Figure 20. For each case, the distribution of $M_{\mathrm{w}}$, which primarily varies between -3.5 and -1.0 , is shown as the histogram (yellow green); the total number of events (i.e., cumulative frequency) is shown by the blue-green dots, which is then used to fit the Gutenburg-Richter law, yielding a $b$-value around 2. Notice the similarities among all three cases in both figures 19 and 20, suggesting that the $b$ value is likely to be independent from the LSDF and poroelastic coupling. We also hypothesize that the breaking-down in the power law distribution of the length of the activated subset of fractures might be responsible for the deviation in the $b$-value for induced seismicity.
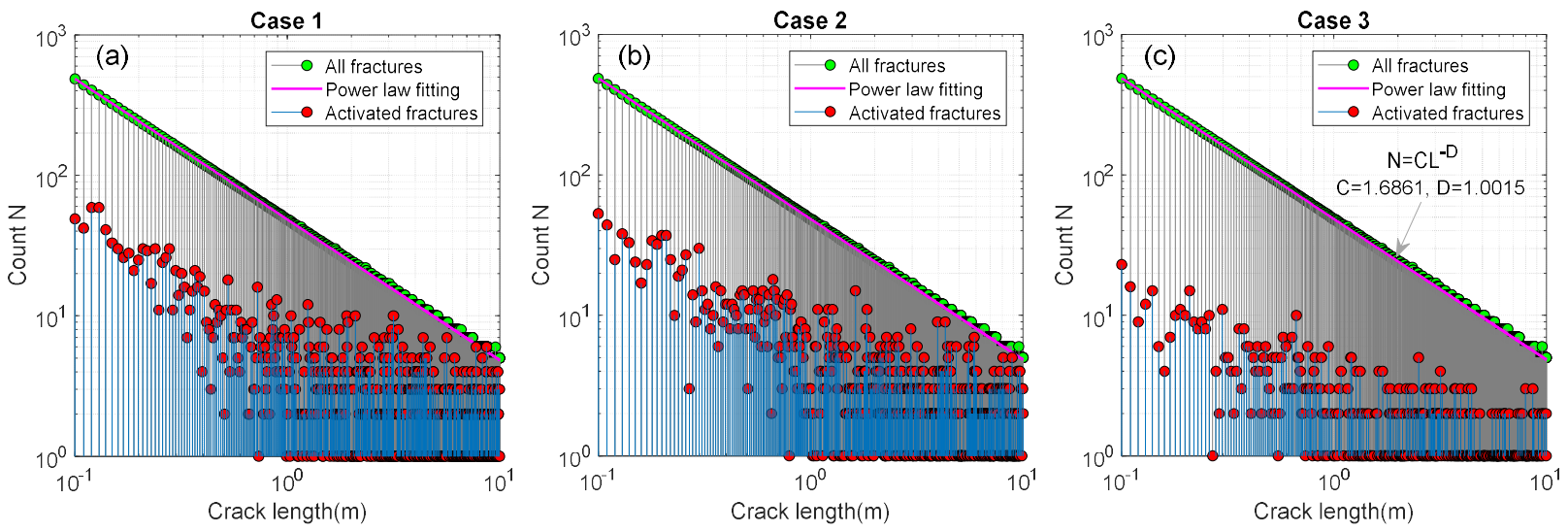

Figure 19. Histogram of the fracture length using 1000 equal-sized bins, plotted on a log-log scale as discrete sequences. The green sequence indicates the distribution of length of all fractures, which follows a power law decay as is fitted with the magenta line. The fitting parameters are also shown, specifically, the fractal dimension $D$ is 1 . The red sequence shows the length distribution of activated fractures only (fractures undergone at least one seismic cycle). Because it is primarily the favorably oriented fractures that are activated, the distribution no longer follows a power law decay. (a)

695 Case 1, (b) case 2 and (c) case 3. 

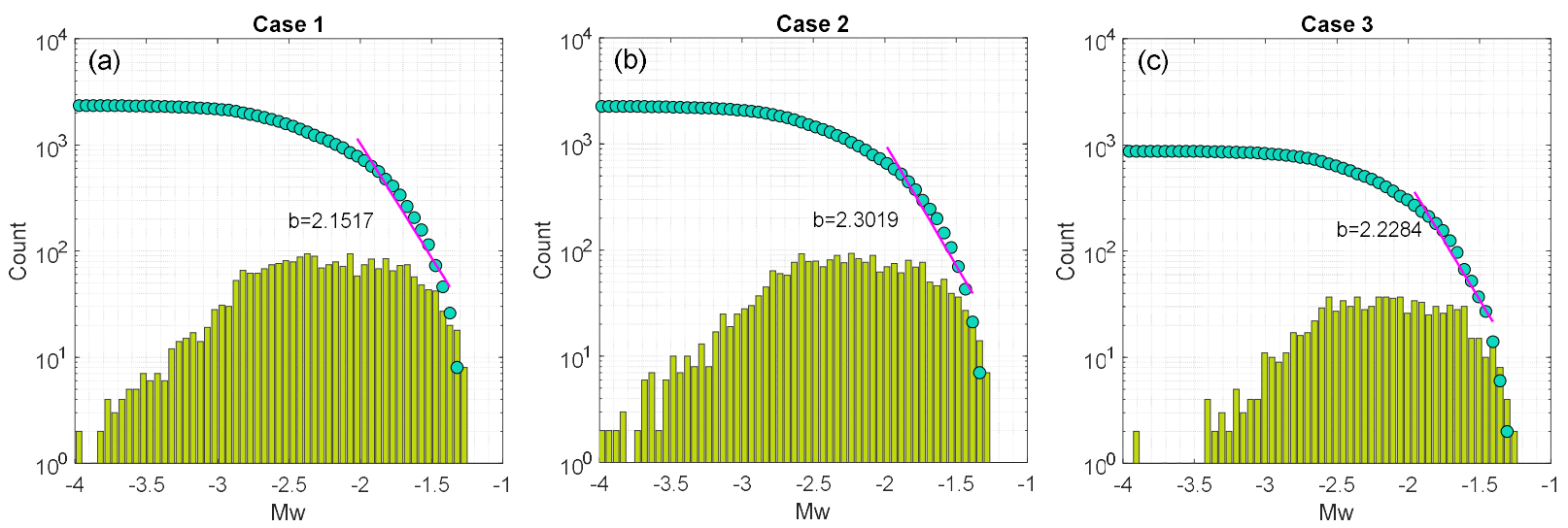

Figure 20. Histogram of the modeled $M_{\mathrm{w}}$ (yellow green). The bin size is 0.05 , and the y-axis is on a log-scale. The associated distribution of $N$ follows the classic Gutenberg-Richter law (blue green); data points with a $M_{\mathrm{w}}$ above -2 are used for fitting (the magenta line), yielding a $b$-value around 2, which is commonly observed for induced seismicity. (a) Case 1, (b) case 2 and (c) case 3.

701 Further, we investigate whether the $b$-value of induced seismicity exhibits spatial or temporal 702 dependences. To do so, for each case, we divide the events into 10 groups in both space and time 703 based on the associated distance $R$ and the origin time $t_{0}$. For each group, we carry out the same $704 b$-value analysis as has been described above and the results are displayed in Figures 21 and 22. 705 In each case, the magnitude-frequency distribution appears alike among all groups in both space and time. The $b$-value is predominantly between 2 and 2.5 and no substantial spatial- or temporaldependence is observed. Such independences are not altered by the LSDF or poroelastic coupling. An exception is shown in Figures 21c and 21f, where the $b$-value is around 3 near the injection and drops to between 2 and 2.5 away from the injection (see also Bachmann et al., 2012), possibly due to some variations among the selected cutoff $M_{\mathrm{w}}$ for data fitting. 

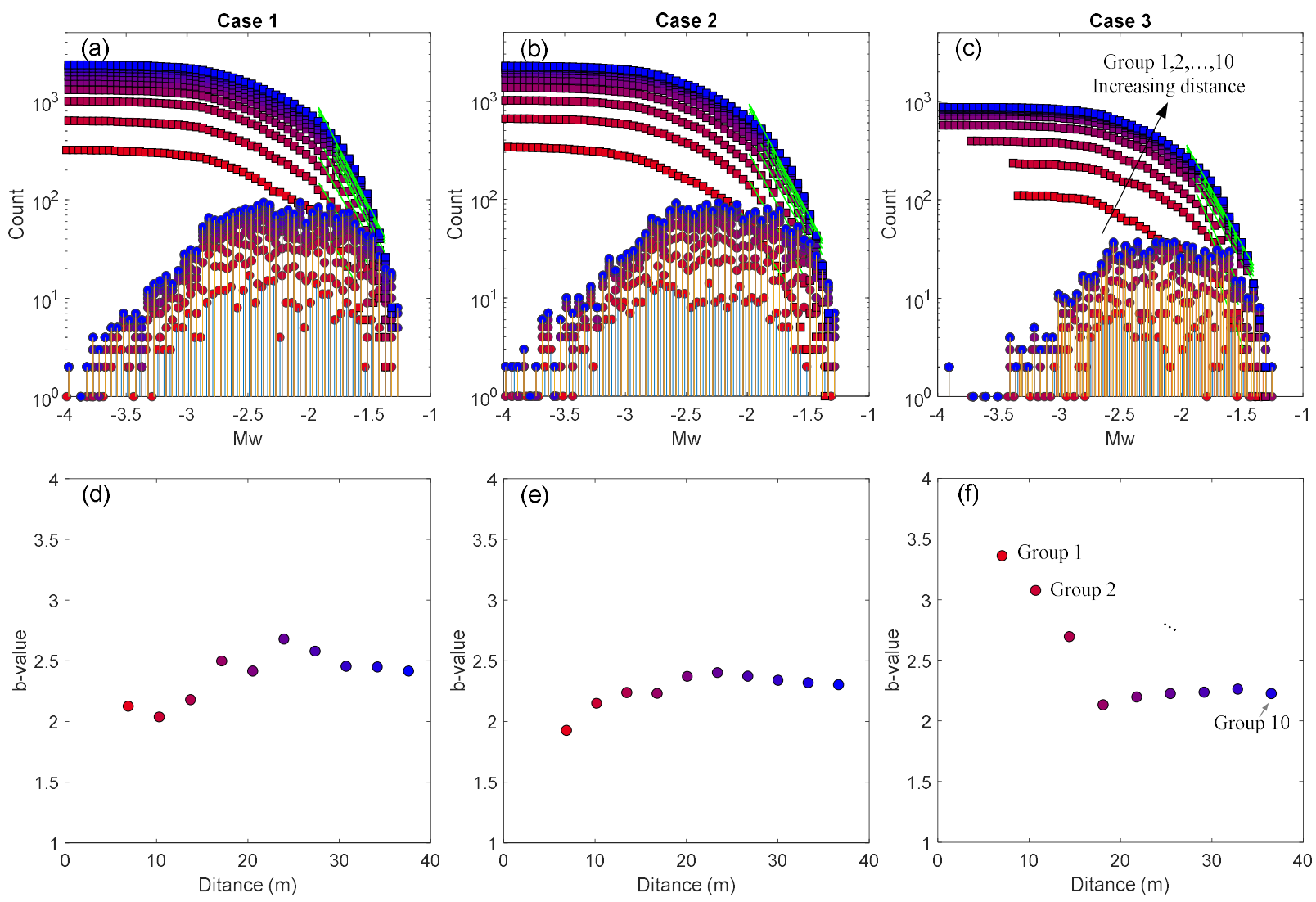

Figure 21. $b$-value analysis in space. The modeled distance interval [ $\left.R_{\min }, R_{\max }\right]$ is divided into 10 equal-sized bins and the events are grouped accordingly. The group number is indicated by the color. A $b$-value is fitted for each group (top row, slope of the green line) and is plotted against the corresponding distance (bottom row). The cutoff $M_{\mathrm{w}}$ for fitting is around 2 but some variations exist among all groups. (a), (d) Case 1, (b), (e) case 2 and (c), (f) case 3. 

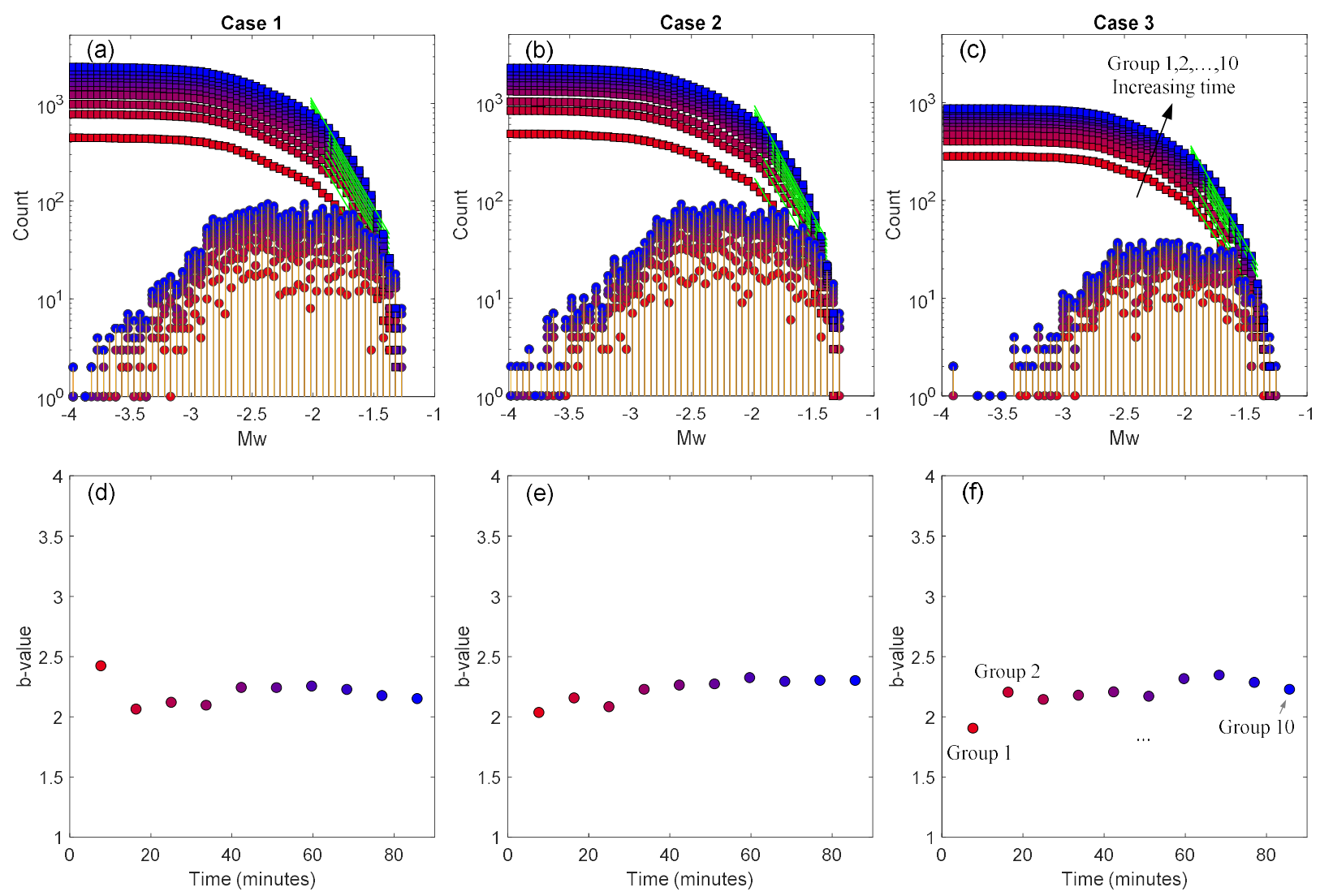

Figure 22. Same as Figure 21 but for the 10 equal-sized origin time intervals on the modeled [ $\left.t_{0 \min }, t_{0 \max }\right]$.

\subsection{Activated Fractures and Permeability Enhancement}

Figure 23 gives four snapshots illustrating the growth of the activated network of fractures for cases 1-3. The network consists of fractures both interconnected to and isolated from the fluid boundary. In the context of unconventional and geothermal reservoir stimulation, the interconnected fractures are indicative of the so-called stimulated reservoir volume and the stimulation efficiency. As can be seen, resolving the LSDF predicts localized permeabilityenhanced flow channels and less area is stimulated as a result. This effect manifests itself if poroelastic coupling is further considered. For each activated fracture, the nondimensionalized permeability changes along directions perpendicular and parallel to it, denoted as $k \perp / k$ and $k / / / k$, respectively, are calculated from the associated $M_{\mathrm{w}}$ using a simple power law scaling relation (appendix A.2). This relation predicts a linear scaling between $k \perp / k$ and $k / / / k$, and therefore both can be normalized into the same quantity $k^{*}$, which indicates the color in Figure 23. As an example, we focus on $(k \perp / k)$ only. For a fracture that has undergone $j$ seismic cycles $(j>1)$ at a time step of interest, $\Sigma_{j}(k \perp / k)$ is calculated as the result at this time step. The modeled maximum $(k \perp / k)$ for a single-event fracture and a multi-event fracture are 30.6 and 81.2 for case 1, 31.1 and 76.7 for case 2 and 30.9 and 49.1 for case 3, suggesting that repeating seismic cycles can further enhance 
734 the permeability by a few more folds compared to just the first seismic cycle but poroelastic coupling seems to counteract this effect.

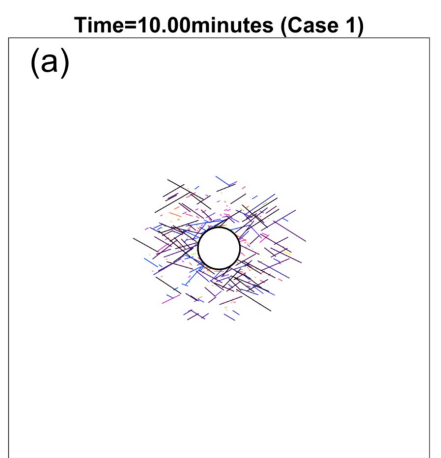

Time=20.00minutes (Case 1)

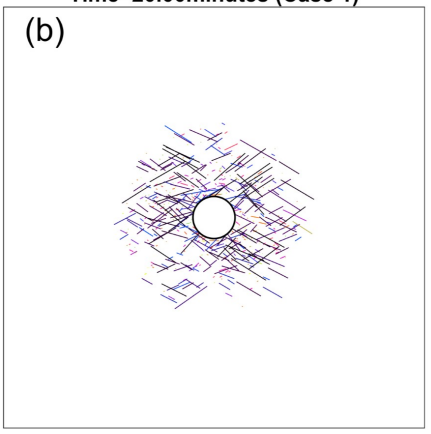

Time $=40.00$ minutes $($ Case 1$)$

(c)

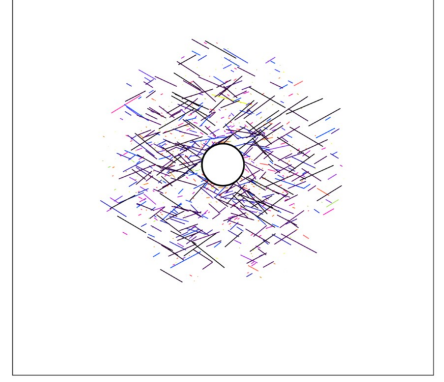

(d)

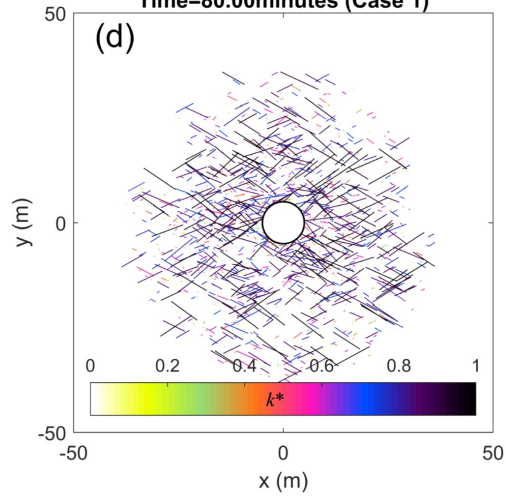

Time $=10.00$ minutes $($ Case 2)

(e)

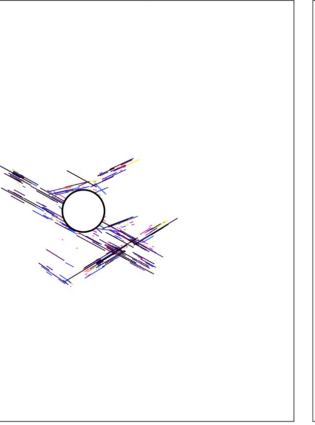

Time=20.00minutes $($ Case 2 )

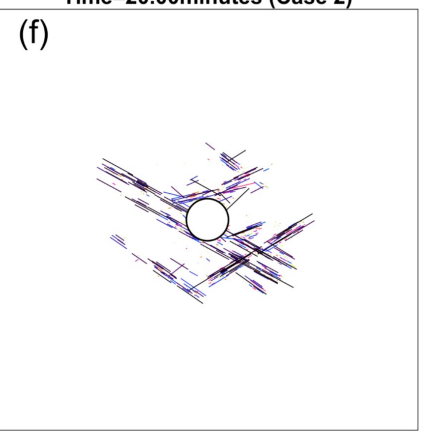

Time=40.00minutes $($ Case 2$)$

(g)

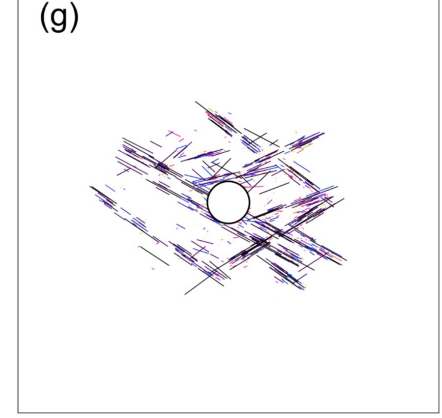

Time=80.00minutes (Case 2)

(h)

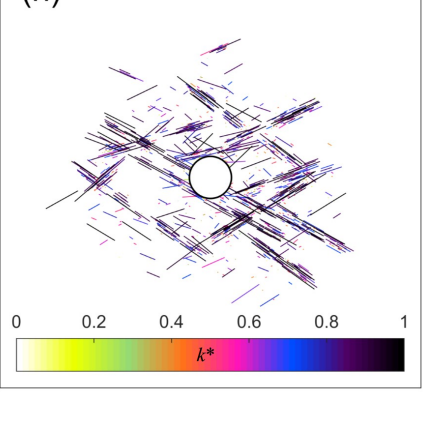

(i)

Time=10.00minutes (Case 3)

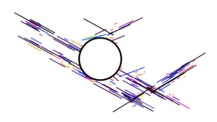

Time=20.00minutes $($ Case 3 )

(j)

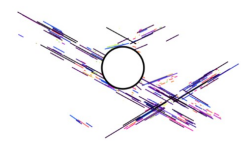

Time $=40.00$ minutes (Case 3$)$

(k)

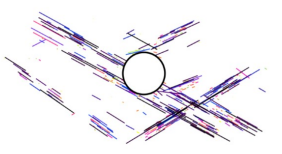

(I)

Time $=80.00$ minutes $($ Case 3 )

(

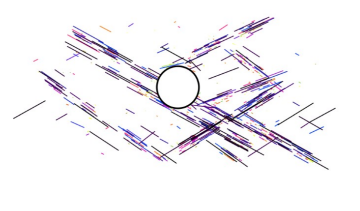

$\begin{array}{lllll}0 & 0.2 & 0.4 & 0.6 & 0.8\end{array}$

Figure 23. Snapshots of the activated fractures at four selected time steps showing the progressive development of the stimulated network. The time is indicated at the top of each plot and the color shows the quantity $k^{*}$ (appendix A.2), which is indicative of the permeability changes along the fracture-normal and -tangential directions. (a)-(d) Case 1, (e)- 
(h) case 2 and (i)-(l) case 3. The differnces between cases 1 and 2 show the effect of the LSDF and the differences between cases 2 and 3 show the effect of poroelastic coupling.

\section{Summary and Conclusions}

We have developed a hydromechanical-stochastic approach to modeling fluid perturbationinduced seismicity in a fluid-saturated and fractured poroelastic medium. Following predefined distributions characteristic of a natural fracture system, we generate a dual network of fractures consisting of large-scale deterministic fractures (LSDF) and small-scale stochastic fractures (SSSF) within the hosting rock. The modeling consists of two sequential steps, including first the quasistatic fracture-poro-mechanical modeling and second the seismicity modeling. In the first step, only the LSDF is considered and it is resolved in a computational model of fluid-solid fully coupled single-phase poromechanics of arbitrarily fractured media. This provides a LSDFcontrolled poroelastic stress tensor as a pivotal input for the second step, in which the complete dual network of fractures is then considered. The seismicity-induced shear stress loss on a slipped fracture is stochastically modeled as a static quantity without explicitly resolving the co-seismic dynamic rupture process; it remains constrained within a range computed from the timedependent poroelastic stress in conjunction with the initial stress and the peak and residual frictional strengths. A prediction-correction type of fracture stress updating scheme is developed accordingly, which naturally produces multiple seismic cycles. Three progressive cases were designed to show the effects of fractures and poroelastic coupling on the resulting seismicity and its characteristics. Compared to the prevalent fracture-free, coupling-free and diffusion-only class of statistical models, our method produces induced seismicity with spatial-temporal characteristics agreeing much better with real data. It also goes beyond the scope of most current models and provides a synthetic catalog of induced events, allowing for the analysis of seismic source characteristics and connections between observations and model physics.

Main findings from this study are:

(1) The spatial-temporal evolution of the pore fluid overpressure $p$, the change in the solid effective stress tensor $\boldsymbol{\sigma}_{p}{ }^{\prime}$ and the associated stress invariants, $I_{1}{ }^{\prime}, \sqrt{J_{2}{ }^{\prime}}$ and $\sqrt{J_{2}{ }^{\prime}}-\sin (\phi) I_{1}{ }^{\prime} / 3$, all differ in a porous medium, a fractured porous medium and a fractured poroelastic medium. In space, the presence of the $L S D F$, if hydraulically conductive, leads to marked localization of these quantities around it and the associated fronts become highly non-smooth. Poroelastic coupling tends to reduce the magnitude of $p$ and $I_{1}{ }^{\prime}$ near fluid-penetrated fractures but predicts an otherwise non-existing $\sqrt{J_{2}{ }^{\prime}}$ within the entire domain. As a result, $\sqrt{J_{2}}{ }^{\prime}-\sin (\phi) I_{1}{ }^{\prime} / 3$ is reduced in the near field but increased in the far field. In the $R-T$ space, $p$ and $I_{1}{ }^{\prime}$ share the same front which is below the front shared by $\sqrt{J_{2}{ }^{\prime}}$ and $\sqrt{J_{2}{ }^{\prime}}-\sin (\phi) I_{1}{ }^{\prime} / 3$.

(2) In space, the LSDF leads to not only heterogeneity but also pronounced clustering in the seismicity. Poroelastic coupling not only enhances the clustering, but also substantially inhibits 
the seismicity and greatly reduces the number of events in the near field. In the far field, although it can remotely trigger some events, its effect does not dominate even in the presences of critically stressed fractures. Overall the event population is significantly reduced. The clustering occurs only near fractures favorably oriented with respect the initial stress tensor $\boldsymbol{\sigma}_{0}{ }^{\prime}$ and meanwhile subjected to sufficient amount of $\sqrt{J_{2}{ }^{\prime}}-\sin (\phi) I_{1}{ }^{\prime} / 3$. Correspondingly, the activated subset of fractures forms permeability-enhanced flow channels localized along the $L S D F$, and this is further manifested by poroelastic coupling. In the $R-T$ space, the characteristics of the seismicity are in good agreement with observations from real data. In addition to heterogeneity, the curvature of the delineated parabolic seismicity front is increased by the LSDF. The state of $\boldsymbol{\sigma}_{0}{ }^{\prime}$ with respect to the fracture peak strength can render the seismicity front lagged behind the $p$ front. A positive correlation is observed between the distance and the origin time for events occurring along the $L S D F$ but not those occurring in the hosting rock.

(3) $\boldsymbol{\sigma}_{p}{ }^{\prime}$ (either coupled or decoupled with $p$ ) and seismicity are the two sources driving changes in the stress on a fracture, and together they can drive the fracture through multiple seismic cycles on a timescale relevant to the problem. This provides a viable mechanism of fluid-induced repeating seismic events characterized with a step-wise stress path. The distribution of the interseismic time between two consecutive repeating events seems independent from both the LSDF and poroelastic coupling. The latter, however, tends to reduce the number of repeating event groups and the number of seismic cycles within a group, in addition to adding nonlinearity to the associated step-wise stress path. Repeating events are also able to increase the permeability change on the fracture by a few folds.

(4) Although collectively referred to as induced seismicity, the modeled events are predominantly triggered as opposed to induced. Because the induced events occur on unfavorably-oriented fractures that require large $p$ or $\sqrt{J_{2}{ }^{\prime}}-\sin (\phi) I_{1}{ }^{\prime} / 3$, they are concentrated near the source of the fluid perturbation.

(5) Some source characteristics of the induced seismicity seem independent from the LSDF and poroelastic coupling. Irrespective of the case, the moment magnitude $M_{\mathrm{w}}$ and by extension, the permeability change $k^{*}$, show similar distributions; the $b$-value varies between 2 and 2.5 and exhibits no substantial space- or temporal-dependence; for the given set of parameters, the stress drop $\Delta \tau$ predominantly falls in between $0.1 \mathrm{MPa}$ and $1 \mathrm{MPa}$, although a higher $\Delta \tau$ is more likely due to the poroelastic medication to the stress path. $\Delta \tau$ generally does not reach the maximum likely stress drop.

(6) In our complete dual fracture system, the length and frequency obey a realistic power law scaling relation; however, this relation no longer holds for the activated subset of fractures, owing to that only favorably-oriented fractures are induced to slip. This might explain the commonly 
811 observed deviation in the $b$-value from around 1 for natural seismicity to around 2 for induced 812 seismicity.

\section{Acknowledgement}

814 We thank Norm Sleep for discussion. Lei Jin is funded by the Stanford Center for Induced and 815 Triggered Seismicity. No data was used in producing this manuscript.

\section{Appendix}

\section{7 \\ A.1 Single-Phase Poromechanics of Arbitrarily Fractured Media}

818 Jin \& Zoback (2017) formulated the problem of single-phase poromechanics of fluid-saturated and arbitrarily fractured porous media. Without presenting the full details, here, we outline several key governing equations. First, the fully coupled mass conservation law and quasi-static force balance law are:

$$
\begin{gathered}
\left(\Lambda_{0}(\underline{x}) \phi_{m 0}(\underline{x})\left(C_{m}+C_{\rho}\right)+\left(1-\Lambda_{0}(\underline{x})\right) \phi_{f 0}(\underline{x})\left(C_{f}+C_{\rho}\right)\right) \dot{p}(\underline{x}, t) \\
-\alpha \nabla \cdot \underline{\dot{u}}(\underline{x}, t)+\nabla \cdot \underline{v}(\underline{x}, t)=s(\underline{x}, t), \quad \underline{x} \in \Omega_{m} \cup \Omega_{f} \\
\nabla \cdot \boldsymbol{\sigma}_{p}^{\prime}(\underline{x}, t)+\alpha \nabla p(\underline{x}, t)=\underline{0}, \quad \underline{x} \in \Omega_{m} \cup \Omega_{f}
\end{gathered}
$$

Next, the two fluid flow equations are given by the Darcy's law and a nonlinear cubic law, designated to the matrix and fractures, respectively. They read:

$$
\begin{gathered}
\underline{v}(\underline{x}, t)=-\eta^{-1} \mathbf{k}_{m}(\underline{x}) \cdot \nabla p(\underline{x}, t), \quad \underline{x} \in \Omega_{m} \\
\underline{v}(\underline{x}, t)=-\eta^{-1} \frac{1}{12}\left(b_{0}\left(1+C_{f} p_{f}(\underline{x}, t)\right)\right)^{2} \nabla_{\tau} p(\underline{x}, t), \quad \underline{x} \in \Omega_{f}
\end{gathered}
$$

Furthermore, the two solid constitutive laws, including a generalized Hooke's law for the hosting rock and a transverse simple shear deformation law for fractures, read:

$$
\begin{gathered}
\boldsymbol{\sigma}_{p}{ }^{\prime}(\underline{x}, t)=\mathbb{C}_{m}: \nabla^{s} \underline{u}_{m}(\underline{x}, t), \quad \underline{x} \in \Omega_{m} \\
\boldsymbol{\sigma}_{p}{ }^{\prime}(\underline{x}, t)=G_{f \tau} \nabla_{n} u_{f \tau}(\underline{\xi}, t), \quad \underline{\xi} \in \Omega_{f}
\end{gathered}
$$

In equations (A1) - (A6), subscripts ' $m$ ' and ' $f$ indicate quantities associated with the hosting rock (porous matrix) and deterministic fractures, subscript ' 0 ' denotes the initial value of a quantity, subscripts ' $n$ ' and ' $\tau$ ' indicate the fracture normal and tangential directions, $\underline{x}$ and $\xi$ indicate the global and fracture local coordinate systems, $t$ is the time, $\Omega$ is the model domain, $\phi$ is the intrinsic porosity, $\Lambda(\underline{\mathrm{x}})$ is a fracture-dependent parameter enabling the definition of a so-called partial porosity, $C$ is the compressibility, $p$ is the fluid overpressure, $\underline{v}$ is the fluid velocity vector, $s$ is the 
external fluid source normalized by the initial fluid density, $\eta$ is the fluid viscosity, $\mathbf{k}$ is the permeability tensor, $b$ is the fracture hydraulic aperture, $\boldsymbol{\sigma}_{p}{ }^{\prime}$ is the solid effective stress (i.e., the poroelastic stress) tensor, $\underline{u}$ is the solid displacement vector, $a$ is the Biot-Willis coefficient, $\mathbf{1}$ is the Kronecker delta, $\mathbb{C}$ is the elastic stiffness tensor under plane strain and $G$ is the fracture shear modulus. $\nabla, \nabla^{s}, \nabla_{n}$ and $\nabla_{\tau}$ are operators for computing the gradient, the symmetric gradient, the fracture-normal gradient and the fracture-tangential gradient, and $\nabla \cdot$ is the divergence operator.

The presence of fractures is reflected in equation (A1) by the modification to the hydraulic storage capacity, and by equations (A4) and (A6) as the augmentation to the hydraulic conductivity and the elastic stiffness of the system. Fracture-induced nonlinearity is introduced by equation (A4) via the pressure-dependent hydraulic aperture. Additionally, by formulating the problem over a single domain, the mass exchange between fractures and the matrix is resolved by, (1) imposing an interface condition in addition to the standard Dirichlet and Neumann boundary conditions, and (2) admitting discontinuities in fracture-normal fluid flux. The model is different from the standard dual-porosity double-permeability model which requires the formulation of two interacting mass conservation laws and the use of a smearing quantity called the 'shape factor' resulting from domain separation and regularization. The initial conditions of the primary unknowns are trivially set up as 0 since we are solving only for the changes.

The fluid diffusion in a fluid-saturated porous medium in the absence of fractures is governed by a simplified version of equation (A1):

$$
\left(\phi_{m 0}(\underline{x})\left(C_{m}+C_{\rho}\right)\right) \dot{p}(\underline{x}, t)+\nabla \cdot \underline{v}(\underline{x}, t)=s(\underline{x}, t), \quad \underline{x} \in \Omega
$$

\section{A.2 Seismic Source Parameters and Scaling Laws}

The key equations used in calculating the seismic source parameters are shown here. First, $M_{0}$ can be calculated from the fracture dimension and the recorded $\Delta \tau$. Depending on the fracture geometry and the faulting regime, various formulas are available. Here, we opt for the one suitable for a rectangular dip-slip fracture (Kanamori and Anderson, 1975):

$$
M_{0}=\frac{\pi(\lambda+2 \mu)}{4(\lambda+\mu)} \Delta \tau W^{2} L
$$

where $W$ is the fracture width (assumed as $1 \mathrm{~m}$ in the numerical examples under plane strain), $\lambda$ and $\mu$ are the Lame's constant and the shear modulus of the medium.

Second, $M_{\mathrm{w}}$ is calculated from $M_{0}$ following (Hanks \& Boore, 1984):

$$
M w=\frac{2}{3}\left(\lg M_{0}-9.1\right)
$$


868

869

870

871

872

873

874

875

876

877

878

879

880

881

882

883

884

885

886

887

888

889

890

891

892

893

894

895

896

Finally, we adopt the following scaling laws that directly relate the permeability changes on a fracture to the event magnitude (Ishibashi et al., 2016):

$$
\begin{aligned}
& k_{\perp} / k=116.4 \times 10^{0.46 M w} \\
& k_{/ /} / k=13.1 \times 10^{0.46 M w}
\end{aligned}
$$

where $\mathrm{k} \perp$ and $\mathrm{k} / /$ are the fracture permeabilities orthogonal and parallel to the fracture, and $k$ is a reference permeability of the fracture prior to slip and is related to the fracture length via a power scaling law. Other methods for mapping permeability changes from induced seismicity data are available (e.g., Fang et al., 2018).

Because of the simple linear relation between $\mathrm{k} \perp$ and $\mathrm{k}_{/ /}$, the normalized permeability changes along the fracture-normal and -tangential directions, denoted as $k_{j}^{*}$ where $\mathrm{j}=\perp$ or $/ /$ and calculated as $\left.k_{j}^{*}=\left(k_{j} / k\right)-\left(k_{j} / k\right)_{\min }\right) /\left(\left(k_{j} / k\right)_{\max }-\left(k_{j} / k\right)_{\min }\right)$, are the same, therefore, both are collectively denoted as $k^{*}$. This quantity is used in section 4.6.

\section{A.3 Definition of Triggered and Induced Events}

Some qualitative definitions of triggered and induced seismicity exist (e.g., McGarr \& Simpson, 1997). Here we propose the following quantitative definition for distinguishing a triggered event from an induced event based on the initial stress on a fracture in relation to the peak and residual frictional strengths:

$$
\begin{array}{r}
\sqrt{\left\|\boldsymbol{\sigma}_{0} \cdot \underline{n}\right\|^{2}-\left(\boldsymbol{\sigma}_{0}{ }^{\prime}: \underline{n} \otimes \underline{n}\right)^{2}} \leq \mu_{d}\left(\boldsymbol{\sigma}_{0}{ }^{\prime}: \underline{n} \otimes \underline{n}\right), \text { induced } \\
\mu_{d}\left(\boldsymbol{\sigma}_{0}{ }^{\prime}: \underline{n} \otimes \underline{n}\right)<\sqrt{\left\|\boldsymbol{\sigma}_{0}^{\prime} \cdot \underline{n}\right\|^{2}-\left(\boldsymbol{\sigma}_{0}{ }^{\prime}: \underline{n} \otimes \underline{n}\right)^{2}} \leq \mu_{s}\left(\boldsymbol{\sigma}_{0}{ }^{\prime}: \underline{n} \otimes \underline{n}\right), \text { triggered }
\end{array}
$$

where $\boldsymbol{\sigma}_{0}{ }^{\prime}, \underline{n}, \mu_{s}$ and $\mu_{d}$ are the same as in the main text.

Equation (A11) states that from a loading point of view, the key difference between the two lies in that an induced event represents shear failure on a fault that is otherwise tectonically inactive with respect to the background stress state, whereas a triggered event is indicative of a fault that is nevertheless expected to produce an earthquake given the background stress state but the process towards failure is favorably accelerated. Our definition is consistent with the aforementioned one. As a result, upon seismicity, a triggered event releases a substantial amount of tectonic stress whereas an induced event releases mostly anthropogenic stress.

\section{A.4 Poroelastic Stress Invariants}

The two poroelastic stress invariants are calculated according to standard formulations except for the use of the effective poroelatic stress tensor $\boldsymbol{\sigma}_{p}{ }^{\prime}$. Under plane strain, they read:

$$
\frac{1}{3} I_{1}{ }^{\prime}=\frac{1}{3}(1+v)\left(\sigma_{p x}^{\prime}+\sigma_{p y}^{\prime}\right)
$$




$$
\sqrt{J_{2}{ }^{\prime}}=\sqrt{\frac{1}{6}\left[\left({\sigma^{\prime}}_{p x}-\sigma_{p y}^{\prime}\right)^{2}+\left(\sigma_{p y}^{\prime}-v\left(\sigma_{p x}^{\prime}+\sigma_{p y}^{\prime}\right)\right)^{2}+\left(\sigma_{p x}^{\prime}-v\left(\sigma_{p x}^{\prime}+\sigma_{p y}^{\prime}\right)\right)^{2}\right]+\left(\sigma_{p x y}^{\prime}\right)^{2}}
$$

898

899

900

901

902

903

904

905

906

907

908

909

910

911

912

913

914 Given the parameters used in this study, specifically, $v=0.25, a=0.8$ and $\mu_{\mathrm{s}}=0.6$, equation (A16) 915 predicts that $I_{1}{ }^{\prime} / 3 \approx-0.67 p$ and $\sqrt{J_{2}{ }^{\prime}}-\sin (\phi) I_{1}{ }^{\prime} / 3 \approx 0.34 p$ for cases 1 and 2.

916

917

918

919

920

921

922

923

924

where $v$ is the Poisson's ratio, $\sigma_{p x}^{\prime}$ and $\sigma_{p y}^{\prime}$ are the two normal components and $\sigma_{p x y}^{\prime}$ is the shear component of $\boldsymbol{\sigma}_{p}{ }^{\prime}$.

Using these two stress invariants, we define an excess poroelastic shear stress invariant denoted as $M C$, which reads:

$$
M C=\sqrt{J_{2}{ }^{\prime}}-\sin (\phi) \frac{1}{3} I_{1}{ }^{\prime}
$$

Here,

$$
\phi=\tan ^{-1}\left(\mu_{s}\right)
$$

Equation (A14) is adapted from the invariant form of the Mohr Coulomb yield function (e.g., Borja, 2013) by setting the cohesion to 0 and the Lode's angle as $\pi / 6$. In a sense, $M C$ is the invariant form of CFF.

For case 3, equations (A12) and (A13) are used to calculate $I_{1}{ }^{\prime} / 3, \sqrt{J_{2}{ }^{\prime}}$ and $\sqrt{J_{2}{ }^{\prime}}-\sin (\phi) I_{1}{ }^{\prime} / 3$ shown in Figure 7. For cases 1 and 2 devoid of the coupling effect, substituting equation (12) into equations (A12) and (A13) yields the following equivalent poroelastic stress invariants (the continuous instead of the discrete fluid pressure is used here):

$$
\frac{1}{3} I_{1}{ }^{\prime}=-\frac{2}{3}(1+v) \alpha p
$$

$$
\sqrt{J_{2}{ }^{\prime}}=0
$$

\section{A.5 Associating Seismicity With The LSDF}

In section 4.2.2, we have pointed out that in cases 2 and 3, a positive correlation between the distance and the origin time can be observed for an explicit event occurring along the LSDF (Figure 3a) but not for an implicit event in the hosting rock (Figure 3b). Here in Figures A1b and A1c, we single out the explicit events in cases 2 and 3 and plot them in the $R-T$ space, and the color indicates the index $I$ of a fracture (equation (8)) with which an explicit event is associated. For case 1, an explicit event cannot be defined; nonetheless, the events on fractures at the same locations (Figure $3 \mathrm{~d}$ ) are plotted and colored with the same $I$ for comparison (Figure A1a). In Figures $\mathrm{A} 1 \mathrm{~b}$ and A1c, the progressive development of events along a deterministic fracture 
becomes evident, i.e., events of the same color delineate a parabolic trend. However, this cannot
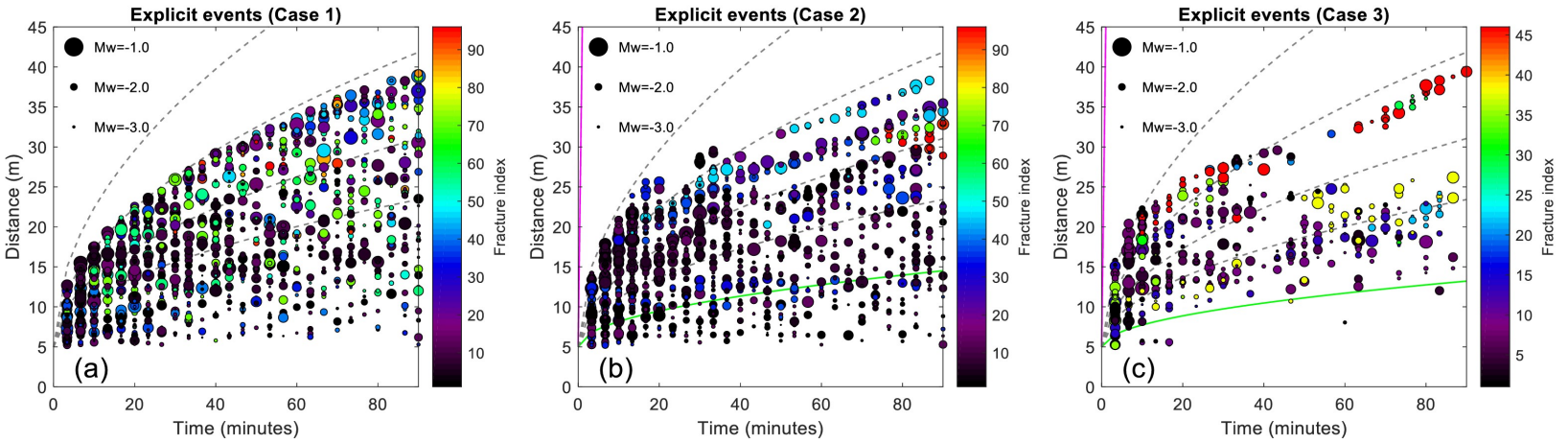

Figure A1. $R$-T plot of the explicit seismic events colored with the associated fracture index $I$. (a) Case 1, (b) case 2 and (c) case 3. Notice that the notion of an explicit event only applied to cases 2 and 3 . Nonetheless, for case 1 , the events at the same locations are plotted for comparison.

\section{A.6 Effect of The Parameter $r$}

932

In section 4.5.1, we have showed the distribution of $\Delta \tau$ in relation to $M_{\mathrm{w}}$ and $L$, which does not vary much among the three cases. The parameter $r$ in equation (18) is generated following a uniform distribution in all cases. Here, to show the effect of $r$, we run a case otherwise identical to case 3 except for the removal of $r$ and the result is shown in Figure A2. While the model produces the same ranges of $M_{\mathrm{w}}$ and $L, \Delta \tau$ is concentrated right above $1 \mathrm{MPa}$. This is not typically observed in real data, implying that $\Delta \tau$ mostly does not reach the maximum likely stress drop.
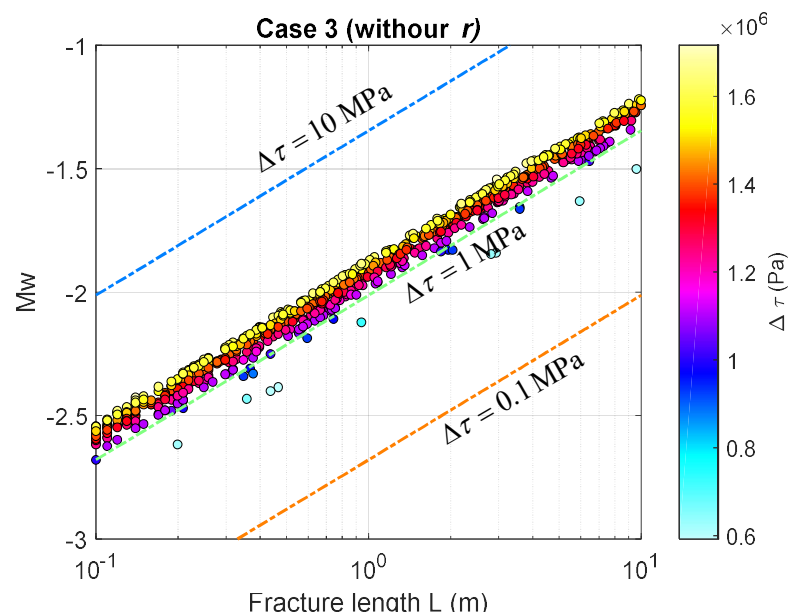

Figure A2. The distribution of $\Delta \tau$ in the $M_{\mathrm{w}}-L$ space for case 3 without considering the random parameter $r$ in equation 940 (18). 


\section{Reference}

942

943

944

945

946

947

948

949

950

951

952

953

954

955

956

957

958

959

960

961

962

963

964

965

966

967

968

969

970

971

972

973

974

975

976

977

978

979

980

981

982

Altmann, J. B., Müller, B. I. R., Müller, T. M., Heidbach, O., Tingay, M. R. P., \& Weißhardt, A. (2014). Pore pressure stress coupling in 3D and consequences for reservoir stress states and fracture reactivation. Geothermics, 52, 195205.

Andrews, D. J. (1976). Rupture velocity of plane strain shear cracks. Journal of Geophysical Research, 81(32), 5679-5687.

Bachmann, C. E., Wiemer, S., Goertz-Allmann, B. P., \& Woessner, J. (2012). Influence of pore-pressure on the event-size distribution of induced earthquakes. Geophysical Research Letters, 39(9).

Baisch, S., \& Harjes, H. P. (2003). A model for fluid-injection-induced seismicity at the KTB, Germany. Geophysical Journal International, 152(1), 160-170.

Baisch, S., Vörös, R., Rothert, E., Stang, H., Jung, R., \& Schellschmidt, R. (2010). A numerical model for fluid injection induced seismicity at Soultz-sous-Forêts. International Journal of Rock Mechanics and Mining Sciences, 47(3), 405-413.

Barbour, A. J., Norbeck, J. H., \& Rubinstein, J. L. (2017). The effects of varying injection rates in Osage County, Oklahoma, on the 2016 M w 5.8 Pawnee earthquake. Seismological Research Letters, 88(4), 1040-1053.

Berkowitz, B. (2002). Characterizing flow and transport in fractured geological media: A review. Advances in Water Resources, 25(8), 861-884.

Biot, M. A. (1941). General theory of three-dimensional consolidation. Journal of applied physics, 12(2), $155-164$.

Block, L. V., Wood, C. K., Yeck, W. L., \& King, V. M. (2015). Induced seismicity constraints on subsurface geological structure, Paradox Valley, Colorado. Geophysical Journal International, 200(2), 1172-1195.

Bonnet, E., Bour, O., Odling, N. E., Davy, P., Main, I., Cowie, P., \& Berkowitz, B. (2001). Scaling of fracture systems in geological media. Reviews of geophysics, 39(3), 347-383.

Borja, R. I. (2013). Plasticity: modeling E computation. Berlin: Springer.

Bruel, D. (2007). Using the migration of the induced seismicity as a constraint for fractured hot dry rock reservoir modelling. International Journal of Rock Mechanics and Mining Sciences, 44(8), 1106-1117.

Byerlee, J. (1978). Friction of rocks. In Rock friction and earthquake prediction (pp. 615-626). Basel: Birkhäuser.

Carcione, J. M., Currenti, G., Johann, L., \& Shapiro, S. (2018). Modeling fluid injection induced microseismicity in shales. Journal of Geophysics and Engineering, 15(1), 234.

Carcione, J. M., Da Col, F., Currenti, G., \& Cantucci, B. (2015). Modeling techniques to study CO2-injection induced micro-seismicity. International Journal of Greenhouse Gas Control, 42, 246-257.

Chang, K. W., \& Segall, P. (2016a). Injection-induced seismicity on basement fractures including poroelastic stressing. Journal of Geophysical Research: Solid Earth, 121(4), 2708-2726.

Chang, K. W., \& Segall, P. (2016b). Seismicity on basement fractures induced by simultaneous fluid injectionextraction. Pure and Applied Geophysics, 173(8), 2621-2636.

Chang, K. W., \& Segall, P. (2017). Reduction of Injection-Induced Pore-Pressure and Stress in Basement Rocks Due to Basal Sealing Layers. Pure and Applied Geophysics, 174(7), 2649-2661.

Deichmann, N., Kraft, T., \& Evans, K. F. (2014). Identification of fractures activated during the stimulation of the Basel geothermal project from cluster analysis and focal mechanisms of the larger magnitude events. Geothermics, 52, 8497.

Deng, K., Liu, Y., \& Harrington, R. M. (2016). Poroelastic stress triggering of the December 2013 Crooked Lake, Alberta, induced seismicity sequence. Geophysical Research Letters, 43(16), 8482-8491.

Duverger, C., Godano, M., Bernard, P., Lyon - Caen, H., \& Lambotte, S. (2015). The 2003 - 2004 seismic swarm in the western Corinth rift: Evidence for a multiscale pore pressure diffusion process along a permeable fracture system. Geophysical Research Letters, 42(18), 7374-7382. 
Fan, Z., Eichhubl, P., \& Gale, J. F. (2016). Geomechanical analysis of fluid injection and seismic fracture slip for the Mw4. 8 Timpson, Texas, earthquake sequence. Journal of Geophysical Research: Solid Earth, 121(4), 2798-2812.

Fang, Y., Elsworth, D., \& Cladouhos, T. T. (2018). Reservoir permeability mapping using microearthquake data. Geothermics, 72, 83-100.

Farmahini-Farahani, M., \& Ghassemi, A. (2016). Simulation of micro-seismicity in response to injection/production in large-scale fracture networks using the fast multipole displacement discontinuity method (FMDDM). Engineering Analysis with Boundary Elements, 71, 179-189.

Goertz-Allmann, B. P., \& Wiemer, S. (2012). Geomechanical modeling of induced seismicity source parameters and implications for seismic hazard assessment. Geophysics, 78(1), KS25-KS39.

Goertz-Allmann, B. P., Goertz, A., \& Wiemer, S. (2011). Stress drop variations of induced earthquakes at the Basel geothermal site. Geophysical Research Letters, 38(9).

Gutenberg, B. (1956). The energy of earthquakes. Quarterly Journal of the Geological Society, 112(1-4), 1-14.

Hanks, T. C., \& Boore, D. M. (1984). Moment-magnitude relations in theory and practice. Journal of Geophysical Research: Solid Earth, 89(B7), 6229-6235.

Hardebol, N. J., Maier, C., Nick, H., Geiger, S., Bertotti, G., \& Boro, H. (2015). Multiscale fracture network characterization and impact on flow: A case study on the Latemar carbonate platform. Journal of Geophysical Research: Solid Earth, 120(12), 8197-8222.

Hirata, T. (1989). A correlation between the b value and the fractal dimension of earthquakes. Journal of Geophysical Research: Solid Earth, 94(B6), 7507-7514.

Hirthe, E. M., \& Graf, T. (2015). Fracture network optimization for simulating 2D variable-density flow and transport. Advances in Water Resources, 83, 364-375.

Hummel, N., \& Shapiro, S. A. (2012). Microseismic estimates of hydraulic diffusivity in case of non-linear fluid-rock interaction. Geophysical Journal International, 188(3), 1441-1453.

Hummel, N., \& Shapiro, S. A. (2013). Nonlinear diffusion-based interpretation of induced microseismicity: A Barnett Shale hydraulic fracturing case study. Geophysics, 78(5), B211-B226.

Ishibashi, T., Watanabe, N., Asanuma, H., \& Tsuchiya, N. (2016). Linking microearthquakes to fracture permeability change: The role of surface roughness. Geophysical Research Letters, 43(14), 7486-7493.

Izadi, G., \& Elsworth, D. (2014). Reservoir stimulation and induced seismicity: Roles of fluid pressure and thermal transients on reactivated fractured networks. Geothermics, 51, 368-379.

Jin, L., \& Zoback, M. D. (2015a). An Analytical Solution for Depletion-induced Principal Stress Rotations In 3D and its Implications for Fracture Stability. In AGU Fall Meeting Abstracts.

Jin, L., \& Zoback, M. D. (2015b). Identification of fracture-controlled damage zones in microseismic data-an example from the Haynesville shale. SEG Technical Program Expanded Abstracts 2015, 726-730.

Jin, L., \& Zoback, M. D. (2016a). Including a stochastic discrete fracture network into one-way coupled poromechanical modeling of injection-induced shear re-activation. In 50th US Rock Mechanics/Geomechanics Symposium. American Rock Mechanics Association.

Jin, L., \& Zoback, M. D. (2016b). Impact of Poro-Elastic Coupling and Stress Shadowing on Injection-Induced Microseismicity in Reservoirs Embedded With Discrete Fracture Networks. In AAPG Annual Convention and Exhibition.

Jin, L., \& Zoback, M. D. (2017). Fully Coupled Nonlinear Fluid Flow and Poroelasticity in Arbitrarily Fractured Porous Media: A Hybrid-Dimensional Computational Model. Journal of Geophysical Research: Solid Earth, 122(10), 76267658.

Jin, L., \& Zoback, M. D. (2018a). Modeling Induced Seismicity: Co-Seismic Fully Dynamic Spontaneous Rupture Considering Fault Poroelastic Stress. In 52nd US Rock Mechanics/Geomechanics Symposium. American Rock Mechanics Association. 
Jin, L., \& Zoback, M. D. (2018b). Fully Dynamic Spontaneous Rupture Due to Quasi-Static Pore Pressure and Poroelastic Effects: An Implicit Nonlinear Computational Model of Fluid-Induced Seismic Events. Journal of Geophysical Research: Solid Earth, 123. https:/ / doi.org/10.1029/2018JB015669

Johann, L., Dinske, C., \& Shapiro, S. A. (2016). Scaling of seismicity induced by nonlinear fluid-rock interaction after an injection stop. Journal of Geophysical Research: Solid Earth, 121(11), 8154-8174.

Johri, M., Zoback, M. D., \& Hennings, P. (2014). A scaling law to characterize fracture-damage zones at reservoir depthsFracture Damage Zones at Depth. AAPG Bulletin, 98(10), 2057-2079.

Kanamori, H., \& Anderson, D. L. (1975). Theoretical basis of some empirical relations in seismology. Bulletin of the seismological society of America, 65(5), 1073-1095.

Keranen, K.M. \& Weingarten, M. (2018). Induced Seismicity. Annual Review of Earth and Planetary Sciences. 46, 149-174.

Ma, X., \& Zoback, M. D. (2017). Laboratory experiments simulating poroelastic stress changes associated with depletion and injection in low-porosity sedimentary rocks. Journal of Geophysical Research: Solid Earth, 122(4), 2478-2503.

Maillot, B., Nielsen, S., \& Main, I. (1999). Numerical simulation of seismicity due to fluid injection in a brittle poroelastic medium. Geophysical Journal International, 139(2), 263-272.

McGarr, Arthur F. and Simpson, David (1997). A broad look at induced and triggered seismicity. In: Rockbrust and seismicity in mines (pp. 385-396). Rotterdam: Balkema.

Megies, T., \& Wassermann, J. (2014). Microseismicity observed at a non-pressure-stimulated geothermal power plant. Geothermics, 52, 36-49.

Moriya, H., Niitsuma, H., \& Baria, R. (2003). Multiplet-clustering analysis reveals structural details within the seismic cloud at the Soultz geothermal field, France. Bulletin of the Seismological Society of America, 93(4), 1606-1620.

Mukuhira, Y., Asanuma, H., Niitsuma, H., \& Häring, M. O. (2013). Characteristics of large-magnitude microseismic events recorded during and after stimulation of a geothermal reservoir at Basel, Switzerland. Geothermics, 45, 1-17.

Mukuhira, Y., Dinske, C., Asanuma, H., Ito, T., \& Häring, M. O. (2017). Pore pressure behavior at the shut-in phase and causality of large induced seismicity at Basel, Switzerland. Journal of Geophysical Research: Solid Earth, 122(1), 411435.

Murphy, S., O’Brien, G. S., McCloskey, J., Bean, C. J., \& Nalbant, S. (2013). Modelling fluid induced seismicity on a nearby active fracture. Geophysical Journal International, 194(3), 1613-1624.

Okubo, P. G., \& Aki, K. (1987). Fractal geometry in the San Andreas fracture system. Journal of Geophysical Research: Solid Earth, 92(B1), 345-355.

Poupinet, G., Ellsworth, W. L., \& Frechet, J. (1984). Monitoring velocity variations in the crust using earthquake doublets: An application to the Calaveras Fracture, California. Journal of Geophysical Research: Solid Earth, 89(B7), 5719-5731.

Raziperchikolaee, S., Alvarado, V., \& Yin, S. (2014). Microscale modeling of fluid flow-geomechanics-seismicity: Relationship between permeability and seismic source response in deformed rock joints. Journal of Geophysical Research: Solid Earth, 119(9), 6958-6975.

Riffracture, J., Dempsey, D., Archer, R., Kelkar, S., \& Karra, S. (2016). Understanding Poroelastic Stressing and Induced Seismicity with a Stochastic/Deterministic Model: an Application to an EGS Stimulation at Paralana, South Australia, 2011. In Proc. 41st Stanford Workshop on Geothermal Reservoir Engineering, SGP-TR-209.

Rothert, E., \& Shapiro, S. A. (2007). Statistics of fracture strength and fluid-induced microseismicity. Journal of Geophysical Research: Solid Earth, 112(B4).

Safari, R., \& Ghassemi, A. (2016). Three-dimensional poroelastic modeling of injection induced permeability enhancement and microseismicity. International Journal of Rock Mechanics and Mining Sciences, 84, 47-58.

Schoenball, M., Davatzes, N. C., \& Glen, J. M. (2015). Differentiating induced and natural seismicity using space-timemagnitude statistics applied to the Coso Geothermal field. Geophysical Research Letters, 42(15), 6221-6228. 
1072

1073

1074

1075

1076

1077

1078

1079

1080

1081

1082

1083

1084

1085

1086

1087

1088

1089

1090

1091

1092

1093

1094

1095

1096

1097

1098

1099

1100

1101

1102

1103

1104

1105

1106

1107

1108

1109

1110

1111

1112

1113

1114

1115

Scuderi, M. M., \& Collettini, C. (2016). The role of fluid pressure in induced vs. triggered seismicity: Insights from rock deformation experiments on carbonates. Scientific reports, 6, 24852.

Segall, P. (1985). Stress and subsidence resulting from subsurface fluid withdrawal in the epicentral region of the 1983 Coalinga earthquake. Journal of Geophysical Research: Solid Earth, 90(B8), 6801-6816.

Segall, P., \& Fitzgerald, S. D. (1998). A note on induced stress changes in hydrocarbon and geothermal reservoirs. Tectonophysics, 289(1), 117-128.

Segall, P., \& Lu, S. (2015). Injection-induced seismicity: Poroelastic and earthquake nucleation effects. Journal of Geophysical Research: Solid Earth, 120(7), 5082-5103.

Segall, P., Grasso, J. R., \& Mossop, A. (1994). Poroelastic stressing and induced seismicity near the Lacq gas field, southwestern France. Journal of Geophysical Research: Solid Earth, 99(B8), 15423-15438.

Shapiro, S. A., Huenges, E., \& Borm, G. (1997). Estimating the crust permeability from fluid-injection-induced seismic emission at the KTB site. Geophysical Journal International, 131(2).

Shapiro, S. A., Rentsch, S., \& Rothert, E. (2005). Characterization of hydraulic properties of rocks using probability of fluid-induced microearthquakes. Geophysics, 70(2), F27-F33.

Shapiro, S. A., Rothert, E., Rath, V., \& Rindschwentner, J. (2002). Characterization of fluid transport properties of reservoirs using induced microseismicity. Geophysics, 67(1), 212-220.

Shi, Y., \& Bolt, B. A. (1982). The standard error of the magnitude-frequency b value. Bulletin of the Seismological Society of America, 72(5), 1677-1687.

Stabile, T. A., Giocoli, A., Perrone, A., Piscitelli, S., \& Lapenna, V. (2014). Fluid injection induced seismicity reveals a NE dipping fracture in the southeastern sector of the High Agri Valley (southern Italy). Geophysical Research Letters, 41(16), 5847-5854.

Stark, M. A., \& Davis, S. D. (1996). Remotely triggered microearthquakes at The Geysers geothermal field, California. Geophysical research letters, 23(9), 945-948.

Tarrahi, M., \& Jafarpour, B. (2012). Inference of permeability distribution from injection-induced discrete microseismic events with kernel density estimation and ensemble Kalman filter. Water Resources Research, 48(10).

Terakawa, T. (2014). Evolution of pore fluid pressures in a stimulated geothermal reservoir inferred from earthquake focal mechanisms. Geophysical Research Letters, 41(21), 7468-7476.

Terakawa, T., Miller, S. A., \& Deichmann, N. (2012). High fluid pressure and triggered earthquakes in the enhanced geothermal system in Basel, Switzerland. Journal of Geophysical Research: Solid Earth, 117(B7).

Terzaghi, Karl (1936). Relation Between Soil Mechanics and Foundation Engineering: Presidential Address. Proceedings, First International Conference on Soil Mechanics and Foundation Engineering, Boston. 3, 13-18.

Verdon, J. P., Stork, A. L., Bissell, R. C., Bond, C. E., \& Werner, M. J. (2015). Simulation of seismic events induced by CO2 injection at In Salah, Algeria. Earth and Planetary Science Letters, 426, 118-129.

Vermylen, J., \& Zoback, M. D. (2011, January). Hydraulic fracturing, microseismic magnitudes, and stress evolution in the Barnett Shale, Texas, USA. In SPE Hydraulic Fracturing Technology Conference. Society of Petroleum Engineers.

Vujević, K., Graf, T., Simmons, C. T., \& Werner, A. D. (2014). Impact of fracture network geometry on free convective flow patterns. Advances in Water Resources, 71, 65-80.

Waldhauser, F., \& Ellsworth, W. L. (2002). Fracture structure and mechanics of the Hayward fracture, California, from double-difference earthquake locations. Journal of Geophysical Research: Solid Earth, 107(B3).

Wassing, B. B. T., Van Wees, J. D., \& Fokker, P. A. (2014). Coupled continuum modeling of fracture reactivation and induced seismicity during enhanced geothermal operations. Geothermics, 52, 153-164.

Yeck, W. L., Weingarten, M., Benz, H. M., McNamara, D. E., Bergman, E. A., Herrmann, R. B., ... \& Earle, P. S. (2016). Far-field pressurization likely caused one of the largest injection induced earthquakes by reactivating a large preexisting basement fracture structure. Geophysical Research Letters, 43(19). 
1116 Yoon, J. S., Zang, A., \& Stephansson, O. (2014). Numerical investigation on optimized stimulation of intact and naturally fractured deep geothermal reservoirs using hydro-mechanical coupled discrete particles joints model. Geothermics, 52, 165-184.

1119 Zbinden, D., Rinaldi, A. P., Urpi, L., \& Wiemer, S. (2017). On the physics-based processes behind production-induced seismicity in natural gas fields. Journal of Geophysical Research: Solid Earth, 122(5), 3792-3812.

1121 Zhao, X., \& Paul Young, R. (2011). Numerical modeling of seismicity induced by fluid injection in naturally fractured reservoirs. Geophysics, 76(6), WC167-WC180.

1123 Zoback, M. D., \& Zinke, J. C. (2002). Production-induced normal fractureing in the Valhall and Ekofisk oil fields. In The Mechanism of Induced Seismicity (pp. 403-420). Basel: Birkhäuser. 